\title{
Close encounters of a rotating star with planets in parabolic orbits of varying inclination and the formation of Hot Jupiters
}

\author{
P. B. Ivanov • J. C. B. Papaloizou
}

Received: date / Accepted: date

\begin{abstract}
In this paper we extend the theory of close encounters of a giant planet on a parabolic orbit with a central star developed in our previous work (Ivanov \& Papaloizou 2004, 2007) to include the effects of tides induced on the central star. Stellar rotation and orbits with arbitrary inclination to the stellar rotation axis are considered. We obtain results both from an analytic treatment that incorporates first order corrections to normal mode frequencies arising from stellar rotation and numerical treatments that are in satisfactory agreement over the parameter space of interest. These results are applied to the initial phase of the tidal circularisation problem. We find that both tides induced in the star and planet can lead to a significant decrease of the orbital semi-major axis for orbits having periastron distances smaller than 5-6 stellar radii with tides in the star being much stronger for retrograde orbits compared to prograde orbits. Assuming that combined action of dynamic and quasistatic tides could lead to the total circularisation of orbits this corresponds to observed periods up to 4-5 days.

We use the simple Skumanich law to characterise the rotational history of the star assuming that the star has its rotational period equal to one month at the age of $5 \mathrm{Gyr}$. The strength of tidal interactions is characterised by circularisation time scale, $t_{e v}$, which is defined as a typical time scale of evolution of the planet's semi-major axis due to tides. This is considered as a func-

P. B. Ivanov

Astro Space Centre, P.N. Lebedev Physical Institute, 4/32 Profsoyuznaya Street, Moscow, 117810, Russia

Tel.: +7-495-3333366

Fax: +7-495-333-2378

E-mail: pbi20@cam.ac.uk

J. C. B. Papaloizou

DAMTP, Centre for Mathematical Sciences, University of Cambridge, Wilberforce Road, Cambridge CB3 0WA, United Kingdom

E-mail: J.C.B.Papaloizou@damtp.cam.ac.uk
\end{abstract}


tion of orbital period $P_{o b s}$, which the planet obtains after the process of tidal circularisation has been completed.

We find that the ratio of the initial circularisation time scales corresponding to prograde and retrograde orbits, respectively, is of order 1.5-2 for a planet of one Jupiter mass having $P_{o b s} \sim$ four days. The ratio grows with the mass of the planet, being of order five for a five Jupiter mass planet with the same $P_{\text {orb }}$. Note, however, this result might change for more realistic stellar rotation histories. Thus, the effect of stellar rotation may provide a bias in the formation of planetary systems having planets on close orbits around their host stars, as a consequence of planet-planet scattering, which favours systems with retrograde orbits. The results reported in the paper may also be applied to the problem of tidal capture of stars in young stellar clusters.

Keywords Tidal interactions · Dynamic tides · Rotating stars · Planet formation and evolution

\section{Introduction}

One explanation for the existence of closely orbiting extrasolar giant planets is through the mechanism of orbital migration. induced through the gravitational interaction with the protostellar disc (e.g. Lin \& Papaloizou 1986). Notably this mechanism is expected to produce planets in circular orbits that are coplanar with the the stellar equator. However, the presence of high orbital eccentricities amongst extra solar planets is indicative of a strong orbital relaxation or scattering process. This led Weidenschilling \& Mazari (1996) and Rasio \& Ford (1996) to consider planet-planet scattering of planets initially on nearby circular orbits that resulted in one planet being scattered onto a highly eccentric orbit that had a close approach to the central star. Subsequent tidal interaction with the central star was then postulated to lead to the formation of a Hot Jupiter. Papaloizou \& Terquem (2001) investigated the strong dynamical relaxation of a planetary system with 5 to 100 planets starting on closely packed circular orbits. This type of relaxation also readily produced planets in a highly eccentric orbits with close enough approaches to the central star for stellar tides to lead to orbital circularisation. The evolution of the planet orbit due to combined action of planet-planet scatterings and tides has also been considered (e.g. Nagasawa et al 2008, Marchi et al 2009).

More recently measurements of the inclination of the orbital planes of transiting protoplanets through the Rossiter-McLaughlin effect has led to the discovery of a significant number of close orbiters on highly inclined or even retrograde orbits (see eg. Winn et al. 2010, Hebrard 2011, and references therein). This indicates that a planet-planet interaction that can lead to high inclination orbits with high eccentricity that are subsequently circularised has been active in some cases at least. Thus tidal circularisation of orbits with arbitrary inclination with respect to the central star need to be considered. Tides induced in the planet are expected to be independent of orbital inclination because of the rapid attainment of pseudo synchronisation (see Ivanov \& Papaloizou 2007). 
However, this is not the case for tides induced on the star. For example in a frame rotating with the star, tidal forcing from a retrograde orbit will appear to have a higher forcing frequency than tidal forcing from a prograde orbit. Accordingly the tidal response will differ. This leads to a larger transfer of energy from the planet orbit to the star during a single periastron flyby (see also Lai 1997) 1 This type of phenomenon may modulate the distribution of observed Hot Jupiters on inclined circular orbits that may have been circularised with tides acting on the central star playing a significant role.

It is the purpose of this paper to study the tidal interactions of planets on highly eccentric orbits, with orbital planes arbitrarily inclined to the stellar equatorial plane, taking account of tides induced on the central star and estimating their contribution to the circularisation process. In making these studies we follow the approach of previous papers (Ivanov \& Papaloizou 2004, 2007; Papaloizou \& Ivanov 2010) and approximate the initial stages of orbital circularisation as being through a sequence of encounters of the planet on a parabolic orbit with the star. We find typical time scales, $t_{e v}$, of evolution of the planet's semi-major axis a due to dynamic tide exerted in the planet and in the star, as a function of the period $P_{o b s}$, which the planet has after the circularisation has been completed and the planet has settled on a low eccentricity orbit. This period can be obtained from observations.

It is important to note that the aspects of dynamical tides considered in this paper can only efficiently decrease the planet's semi-major axis when either the so-called stochastic instability (see e.g. Kochanek (1992), Kosovichev \& Novikov (1992), Mardling (1995)a,b, Ivanov \& Papaloizou (2004), (2007)) operates in the dynamical system consisting of the orbit and tidally perturbed normal modes of the planet and the star, or when the normal modes are able to dissipate the energy transferred from the orbit during a time of order of or smaller than one orbital period. As we discuss below both processes can be effective when the semi-major axis of the orbit is sufficiently large, of the order of several au. Therefore, in general, the time $t_{e v}$ characterises only the orbital evolution at such scales. At smaller scales, nonlinear effects not considered here, such as wave breaking, or frictional processes acting on quasistatic tides can further decrease $a$ thus completing the process of circularisation and leading to the formation of close-in giant planets with small $P_{o b s}$ and eccentricities (e.g. Barker \& Ogilvie 2009 and references therein). Provided that typical time scale of orbital circularisation due to these processes is smaller than $t_{e v}$, this characteristic time scale may be considered as giving an overall time for the planet to circularise from an initially large semi-major axis $a$. In the opposite limit the overall time scale is obviously given by the time scale associated with the later evolution.

In order to evaluate $t_{e v}$ we have to know the dependence of the stellar angular velocity, $\Omega$, on time $t$. In what follows we use the simplest possible

1 As far as we are aware, Lai was the first to point out that energy transfer during a flyby on a retrograde orbit is enhanced compared to the prograde case. In this work the orbital plane was taken to coincide with the equatorial plane. Here we allow for the general case of arbitrary mutual orientations of these planes. 
form of such a dependence assuming the so-called the Skumanich (1972) law for stellar rotation $\Omega \propto t^{-1 / 2}$, where the coefficient of proportionally is chosen in such a way that the star has a period of rotation of one month at an age of 5 Gyr corresponding to that of the Sun.

We find $t_{e v}$ to be different for prograde and retrograde orbits, with the difference increasing with planet mass. The ratio of the evolution time scale corresponding to a prograde orbit to that corresponding to a retrograde orbit is of order $1.5-2$ for a one Jupiter mass planet with a typical $P_{o b s} \sim 4 d$, and it is of order 5 for a five Jupiter mass planet with the same period. This difference gets more pronounced in a situation where tides exerted in the planet are not taken into account. Since conditions determining the long term effectiveness of the transfer of energy from the orbit to modes of pulsation can be different for the planet and star, such a situation may indeed occur. The results reported in this paper may be directly used in numerical experiments involving planetplanet scattering which also include tidal interactions between planets and the host star.

The plan of the paper is as follows. We develop an analytic theory for calculating the tidal response of a slowly rotating star due to close the passage of a planetary perturber in a parabolic orbit in section 2. The orbital plane is allowed to have an arbitrary inclination to the stellar equatorial plane and corrections to stellar normal mode frequencies correct to first order in the stellar angular velocity are taken into account. We give expressions for the energy transferred to the star in section 3 and the analogous expressions for the transferred angular momentum in Appendix B. In section 4 we describe numerical solutions of the linear tidal encounter problem. Following our previous work (Papaloizou \& Ivanov 2010) we take account of Coriolis forces, but not centrifugal forces so that the tidally undisturbed model was spherical. However, apart from this, the stellar angular velocity may take any value. We perform calculations for a polytrope of index $n=3$ and unlike in our previous work we include a treatment of the self-gravity of the star.

Results for the energy and angular momentum transferred to the star as a consequence of prograde encounters, retrograde encounters and encounters with orbital plane perpendicular to the equatorial plane are then given in section 5. We then go on to compare numerical and analytic results in section 6 . In both approaches the tidal transfers are significantly larger for retrograde encounters as compared to prograde encounters. We use our results to estimate the initial time scale for tidal circularisation for an orbit with an initial semi-major axis of 10au that circularises in a short period orbit in section 6.2 for planets of one and five Jupiter masses. We give estimates with and without contributions arising from tides induced in the planet obtained from our previous work (eg. Ivanov \& Papaloizou 2007). The central star is taken to be a solar mass star with angular velocity determined by the Skumanich (1972) law. We find that tides induced in the star and planet may separately account for the initial circularisation of orbits down to periods of a few days. Finally in section [7] we summarize and our discuss our results and their application to the tidal circularisation of prograde and retrograde Hot Jupiters. 
2 The parabolic encounter of a planet with a rotating star: Analytic development for slow rotation and orbits of arbitrary inclination

We develop the theory of the tidal interaction of a planet on a parabolic orbit with a central star. When the star is not rotating the tidal interaction excites $p, f$ and $g$ modes. A slow rotation approximation is then adopted in which the first order corrections to the mode frequencies due to rotation is taken into account in calculating the tidal response for an orbit with arbitrary inclination.

\subsection{Coordinate systems}

We calculate the energy transfer occurring as a result of the action of dynamic tides induced in a rotating star by a planet moving in a highly eccentric orbit in a plane that is inclined with respect to the stellar equatorial plane. The eccentricity is taken to be very close to unity so that to a good approximation the planet may be viewed as undergoing a sequence of parabolic encounters with the central star.

For such a configuration, there are two natural Cartesian coordinate systems with origin at the centre of the star. The first, $\left(X^{\prime}, Y^{\prime}, Z^{\prime}\right)$, is such that the $\left(X^{\prime}, Y^{\prime}\right)$ plane coincides with the orbital plane. The second, $(X, Y, Z)$, is such that the $(X, Y)$ plane coincides with the equatorial plane of the star. It is assumed that the the line of nodes which coincides with the $\mathrm{Y}$ axis makes an angle $\gamma$ with the $Y^{\prime}$ axis and lies in the interval $[0,2 \pi]$. The direction of orbital motion is assumed to be correspond to a rotation about the $Z^{\prime}$ axis when viewed in the $\left(X^{\prime}, Y^{\prime}\right)$ plane with the apsidal line coinciding with the $X^{\prime}$ axis and pericentre such that $X^{\prime}>0$. Thus the $\left(X^{\prime}, Y^{\prime}\right)$ plane coincides with the orbital plane with the orbit being symmetric with respect to the $X^{\prime}$ axis. The $(X, Y)$ plane coincides with the star's equatorial plane with the $Z$ axis directed along the star's rotation axis. The line of intersection of the $\left(X^{\prime}, Y^{\prime}\right)$ plane and the $(X, Y)$ plane is chosen to define the $Y$ axis.

The angle $\beta$ between the $Z^{\prime}$ ) and $Z$ axes is called the inclination angle. Its values lie in the range $[0, \pi]$. Clearly, $\beta=\pi$ corresponds to a situation when the planet's orbit lies in the equatorial plane of the star but the direction of orbital motion is opposite to the direction of stellar rotation. It is easy to see that the mutual orientation of the coordinate systems defined in this way corresponds to the angles $\beta$ and $\gamma$ being the usual Euler angles that are adopted to define the relative orientation of two coordinate systems2. Additionally, we make use of two spherical polar coordinate systems with spherical angles $\left(\theta^{\prime}, \phi^{\prime}\right)$ and $(\theta, \phi)$ respectively. The pairs of angles $\left(\theta^{\prime}, \phi^{\prime}\right)$ and $(\theta, \phi)$ are defined in

\footnotetext{
2 Note that the definition of the Euler angles correspond to the so-called zyz convention. Another way of defining the angles would be to perform the first rotation about the $X$ axis and the second one about the $Z$ axis. The so-defined Euler angles $\beta^{\prime}$ and $\gamma^{\prime}$ correspond to the frequently used $z x z$ convention. They are related to the angles used in this paper as $\beta^{\prime}=\beta$ and $\gamma^{\prime}=\gamma-\pi / 2$.
} 
the usual way, but with respect to the coordinate systems $\left(X^{\prime}, Y^{\prime}, Z^{\prime}\right)$ and $(X, Y, Z)$, respectively.

\subsection{The tidal potential}

The quantity that determines the energy and angular momentum transferred between planet and star is the tidal potential, $U$. When the $\left(X^{\prime}, Y^{\prime}, Z^{\prime}\right)$ system is used the tidal potential has the standard form (see e.g. Press \& Teukolsky 1977, hereafter PT)

$$
U=\frac{G M r^{2}}{R^{3}(t)} \sum_{m}^{\prime} W_{m} e^{-i m \Phi(t)} Y_{2, m}\left(\theta^{\prime}, \phi^{\prime}\right),
$$

where $G$ is the gravitational constant, $M$ is the planet's mass and $r$ is the spherical polar radial coordinate. $R(t)$ and $\Phi(t)$ are the distance between the planet and the star and the angle between the position vector of the planet and the $X^{\prime}$ axis measured in the orbital plane respectively. The time $t=0$ corresponds to a passage through periastron by the planet. The prime above the summation sign implies hereafter that the summation is performed over $m=0, \pm 2$ only. For $|m|=2, W_{m}=\sqrt{\frac{3 \pi}{10}}$ and $W_{0}=-\sqrt{\frac{\pi}{5}}$, with $Y_{l, m}$ being the usual spherical functions.

When we consider the tidal potential in the $\left(X^{\prime}, Y^{\prime}, Z^{\prime}\right)$ coordinate system with orientation determined by the stellar rotation axis, its form is modified. In order to find the new form of $U$ we can use the transformation law of spherical functions under a rotation of the coordinate system. This may be written

$$
Y_{2, m}\left(\theta^{\prime}, \phi^{\prime}\right)=e^{-i m \gamma} \sum_{n} d_{n, m}^{(2)} Y_{2, n}(\theta, \phi),
$$

where $d_{n, m}^{(2)}$ are the so-called Wigner $d$ functions, see e.g. Varshalovich et al (1989). Here the summation is performed over all integers, $n$, such that $n \in$ $[-2,2]$. Note that the $d$ functions depend only on the inclination angle, $\beta$, and satisfy the condition $d_{n,-m}^{(2)}=(-1)^{n-m} d_{-n, m}^{(2)}$. Their explicit forms are given in Appendix A.

Substituting equation (2) in (1) we get

$$
U=r^{2} \sum_{n} A_{n} Y_{2, n}(\theta, \phi),
$$

where

$$
A_{n}=\frac{G M}{R(t)^{3}} \sum_{m}^{\prime} W_{m} e^{-i m(\gamma+\Phi(t))} d_{n, m}^{(2)} .
$$

Note that from the requirement that $U$ is a real quantity and the properties of the spherical functions that $Y_{2, n}^{*}=(-1)^{n} Y_{2,-n}$, where $(*)$ stands for the complex conjugate it follows that

$$
A_{n}^{*}=(-1)^{n} A_{-n} .
$$


In order to evaluate the effects of a single encounter of the planet with the star, we introduce the Fourier transform in time of any quantity of interest, say $F(t), \tilde{F}(\sigma)$, by the relations

$$
F(t)=\int_{-\infty}^{+\infty} d \sigma \exp (-i \sigma t) \tilde{F}(\sigma), \quad \tilde{F}(\sigma)=\frac{1}{2 \pi} \int_{-\infty}^{+\infty} d t \exp (i \sigma t) F(t) .
$$

From equations (5) and (6) it follows that the Fourier transforms of the amplitudes $A_{n}$ obey the symmetry relations

$$
\tilde{A}_{n}^{*}(-\sigma)=(-1)^{n} \tilde{A}_{-n}(\sigma) .
$$

As follows from equations (11) and (3) the time dependence of the tidal potential is determined by the quantities $I_{m}=\left(R_{m i n} / R(t)\right)^{3} e^{-i m \Phi(t)}$, where $R_{\text {min }}$ is the periastron distance. Assuming that the orbit is formally parabolic, $R(t)$ and $\Phi(t)$ can be obtained from the parametric representations

$$
\begin{gathered}
\frac{R}{R_{\text {min }}}=1+x^{2} \\
\Phi=2 \tan ^{-1} x \\
\Omega_{\text {orb }} t=\sqrt{2}\left(x+\frac{x^{3}}{3}\right) \quad-\infty<t<\infty
\end{gathered}
$$

Using these the Fourier transforms of these quantities, $\tilde{I}_{m}$, can be readily obtained in the form

$$
\tilde{I}_{m}=\frac{\sqrt{2}}{\pi \Omega_{\text {orb }}} I_{2,-m}\left(\sigma \Omega_{\text {orb }}^{-1}\right),
$$

where

$$
\Omega_{\text {orb }}=\sqrt{(1+q) \frac{G M_{*}}{R_{m i n}^{3}}}
$$

it a characteristic angular frequency of periastron passage, $M_{*}$ is the mass of the star, $q=M / M_{*} \ll 1$ is the mass ratio, and

$$
I_{2, m}(y)=\int_{0}^{\infty} \frac{d x}{\left(1+x^{2}\right)^{2}} \cos \left(\sqrt{2} y\left(x+x^{3} / 3\right)+2 m \arctan x\right),
$$

see e.g. PT 3 . Using equations (4) and the definition of $\tilde{I}_{m}$ we obtain as explicit expression for $\tilde{A}_{n}(\sigma)$,

$$
\tilde{A}_{n}(\sigma)=\frac{\sqrt{2}}{\pi \Omega_{o r b}} \frac{G M}{R_{m i n}^{3}} a_{n}, \quad a_{n}=\sum_{m}^{\prime} W_{m} e^{-i m \gamma} d_{n, m}^{(2)} I_{2,-m} .
$$

3 Note a misprint in Ivanov \& Papaloizou (2007). In their equation (61) equivalent to (13) there should be $I_{2, m}$ on the left hand side instead of $I_{2,-m}$ as in the paper. 


\subsection{Equations of motion}

As has been discussed in our previous papers (Ivanov \& Papaloizou 2004, Papaloizou\&Ivanov 2005, Ivanov\&Papaloizou 2007, Ivanov \& Papaloizou 2010, Papaloizou\&Ivanov 2010) for the case of coplanar encounters a perturber moving on eccentric orbit excites normal modes of a rotating body after the periastron passage. We must, accordingly, solve equations of motion of stellar perturbations associated with the modes under the forcing determined by the tidal potential in order to find the transfers of energy and angular momentum from the orbit to the modes. As we discuss above in this paper we would like to relax the assumption of coplanarity of the orbital and equatorial planes treating, however, the effect of stellar rotation in a simplest possible way and assuming that the angular frequency of the star, $\Omega$, is much smaller that angular frequencies of the normal modes, $\omega_{n m}$, mainly excited in course of the tidal encounter, $\Omega \ll \omega_{n m}$. In this case the normal modes frequencies are close to the ones of non-rotating star, $\omega_{a}$, where the index $a$ labels different types of normal modes of a non-rotating star (e.g. p-modes, g-modes and the fundamental mode). In the linear approximation in small parameter $\Omega / \omega_{a}$ only the modes frequencies acquire corrections of order of $\Omega$ due to rotation while other quantities remain the same as in the non-rotating case. In the same approximation the total energy and angular momentum transfers can be represented as a direct sum of partial contributions corresponding to modes of different type, and, therefore, we derive below these expressions for a mode of one particular type, omitting, for simplicity, the index $a$ below, thus e.g. $\omega \equiv \omega_{a}$, etc.

The stellar perturbations can be described in terms of the Lagrangian displacement vector, $\boldsymbol{\xi}(t, \mathbf{r})$. Since the tidal potential is represented as a sum of the spherical harmonics $Y_{l, n}$ having $l=2$ and $-2 \leq n \leq 2$ (see equation (3)) in order to solve the response problem for a mode having in the non-rotating limit a frequency $\omega$ and $l=2$ we should consider the vector $\boldsymbol{\xi}(t, \mathbf{r})$ as a linear combination of the eigen vectors with the same values of $n$,

$$
\boldsymbol{\xi}=\sum_{n} b_{n}(t) \boldsymbol{\xi}_{n}(\mathbf{r})
$$

where

$$
\boldsymbol{\xi}_{n}(\mathbf{r})=\xi_{r}(r) Y_{2, n} \mathbf{e}_{r}+\xi_{s}(r)\left(r \nabla Y_{2, n}\right),
$$

$\mathbf{e}_{r}$ is unit vector in the radial direction, the functions $\xi_{r}$ and $\xi_{s}$ can be found by solving the eigenvalue problem for a non-rotating star, see e.g. PT, Lee \& Ostriker 1986. It is assumed hereafter that the eigen-vectors of the star are normalised by the condition (PT)

$$
\int d^{3} x \rho\left(\boldsymbol{\xi}_{n}^{*} \cdot \boldsymbol{\xi}_{n}\right)=\int_{0}^{R_{*}} r^{2} d r \rho\left(\xi_{r}^{2}+6 \xi_{s}^{2}\right)=1,
$$

where integration is performed over the volume of the star, $\rho$ is the stellar density. 
Equations of motion for the coefficients $b(t)$ can be found e.g. in Ivanov \& Papaloizou (2004) as

$$
\ddot{b}_{n}+\omega_{0}^{2} b_{n}+2 i \omega_{n}^{1} \dot{b}_{n}=S, \quad \text { with } \quad S=\int d^{3} x \rho\left(\boldsymbol{\xi}_{n}^{*} \cdot \nabla U\right),
$$

where for a normal mode with time dependence through a factor $\exp (-\mathrm{i} \omega t)$, we set $\omega=\omega_{0}+\omega_{n}^{1}$, with $\omega_{0}$ being the eigenfrequency for the non rotating star, and the frequency correction due to rotation of the star is $\omega_{n}^{1}$, which can be represented as (see e.g. Christensen-Dalsgaard 1998)

$$
\omega_{n}^{1}=n \beta_{r} \Omega
$$

the values of the coefficient $\beta_{r} \sim 1$ were calculated by Saio (1981) for a number of $l=2$ normal modes of a $n=3$ polytropic star.

Substituting equation (3) in the expression for the source term $S$ in (18) we get

$$
S=A_{n} Q, \quad Q=\int_{0}^{R_{*}} r^{3} d r \rho\left(2 \xi_{r}+6 \xi_{s}\right) .
$$

The overlap integrals $Q$ have been calculated by PT and Lee \& Ostriker 1986 for a number of polytropic stellar models.

From equation (18) it follows that the Fourier transform of the amplitude $b_{n}, \tilde{b}_{n}$, can be expressed through the Fourier transform of the amplitude $A_{n}$ defined in equation (3), $\tilde{A}_{n}$, as

$$
\tilde{b}_{n}=\frac{Q \tilde{A}_{n}}{D(\sigma)}, \quad D(\sigma)=\omega_{0}^{2}-\left(\sigma+i \nu-\omega_{2}^{1}\right)^{2},
$$

where we add a small imaginary correction to $\sigma, \sigma \rightarrow \sigma+i \nu, \nu>0$ according to the Landau prescription and neglected $\left(\omega_{2}^{1}\right)^{2}$ as a small quantity.

\section{The energy transfer}

During the planet's flyby of the star the energy is deposited in the star at a rate determined by work done by the tidal force

$$
\dot{E}=\int d^{3} x \rho(\dot{\xi} \cdot \nabla U)
$$

and, accordingly, the total energy transfer, $\Delta E$, is obtained by integration of (22) over time

$$
\Delta E=\int_{-\infty}^{+\infty} d t \int d^{3} x \rho(\dot{\xi} \cdot \nabla U),
$$

where the limits of integration are formally extended to $\pm \infty$ since the integral over time in (23) is highly peaked near $t=0$ corresponding to the periastron passage. 
We substitute equations (3), (15) and (16) in (23) and integrate over the volume of the star using the known orthogonality properties of the spherical functions and relations (5) to get

$$
\Delta E=Q \sum_{n} \int_{-\infty}^{+\infty} d t \dot{b}_{n} A_{n}^{*}
$$

where we recall that the overlap integral $Q$ is given by (20). Now we express $\dot{b}_{n}$ and $A_{n}$ in (23) in terms of their respective Fourier transforms using (6) and integrate the result over time with help of the well known property that $\int d t e^{-i t\left(\sigma-\sigma^{\prime}\right)}=2 \pi \delta\left(\sigma-\sigma^{\prime}\right)$ to obtain

$$
\Delta E=-2 \pi i Q^{2} \sum_{n} \int_{-\infty}^{\infty} d \sigma \sigma \frac{\left|\tilde{A}_{n}\right|^{2}}{D(\sigma)}
$$

where $D(\sigma)$ is defined in equation (21).

A simple analysis shows that in the limit of $\nu \rightarrow 0$ the integral in (25) is mainly determined by contributions close to the poles of the expression under the integral, where $D(\sigma) \approx 0$ and, accordingly,

$$
\sigma \approx \omega_{n}^{ \pm} \equiv \pm \omega_{0}+\omega_{n}^{1}
$$

Taking into account only these contributions in (25) we get

$$
\Delta E=(\pi Q)^{2} \sum_{n}\left(1+\frac{\omega_{n}^{1}}{\omega}\right)\left|\tilde{A}_{n}\left(\omega_{n}^{+}\right)\right|^{2}+\left(1-\frac{\omega_{n}^{1}}{\omega}\right)\left|\tilde{A}_{n}\left(\omega_{n}^{-}\right)\right|^{2} .
$$

It is convenient to use only positive values of the resonant frequencies in (27). Taking into account that $\omega_{-n}^{1}=-\omega_{n}^{1}$ and, accordingly $\omega_{n}^{-}=-\omega_{-n}^{+}$, and using equation (7) we have

$$
\Delta E=(\pi Q)^{2} \sum_{n}\left(1+\frac{\omega_{n}^{1}}{\omega}\right)\left|\tilde{A}_{n}\left(\omega_{n}^{+}\right)\right|^{2}+\left(1-\frac{\omega_{n}^{1}}{\omega}\right)\left|\tilde{A}_{-n}\left(\omega_{-n}^{+}\right)\right|^{2} .
$$

Now we substitute equation (14) in (28) to obtain

$$
\Delta E=4\left(\frac{G M Q}{\Omega_{\text {orb }} R_{\text {min }}^{3}}\right)^{2} \epsilon
$$

where

$$
\begin{gathered}
\epsilon=\left|a_{0}(\omega)\right|^{2}+\left(1+\frac{\omega_{1}^{1}}{\omega}\right)\left|a_{1}\left(\omega_{1}^{+}\right)\right|^{2}+\left(1-\frac{\omega_{1}^{1}}{\omega}\right)\left|a_{-1}\left(\omega_{-1}^{+}\right)\right|^{2} \\
+\left(1+\frac{\omega_{2}^{1}}{\omega}\right)\left|a_{2}\left(\omega_{2}^{+}\right)\right|^{2}+\left(1-\frac{\omega_{2}^{1}}{\omega}\right)\left|a_{-2}\left(\omega_{-2}^{+}\right)\right|^{2},
\end{gathered}
$$

and we take into account that the terms with opposite signs of $n$ give the same contribution in the summation series in (28). Using equation (14) we can also 
obtain explicit expressions for the quantities $\left|a_{n}(\omega)\right|^{2}$ entering in (30) in the form

$$
\begin{aligned}
& \left|a_{n}(\omega)\right|^{2}=W_{2}^{2}\left(d_{n, 2}^{2} I_{2,-2}^{2}+d_{n,-2}^{2} I_{2,2}^{2}+2 d_{n, 2} d_{n,-2} I_{2,-2} I_{2,2} \cos 4 \gamma\right) \\
& \quad+2 W_{2} W_{0} d_{n, 0} I_{2,0} \cos 2 \gamma\left(d_{n, 2} I_{2,-2}+d_{n,-2} I_{2,2}\right)+W_{0}^{2} d_{n, 0}^{2} I_{2,0}^{2}
\end{aligned}
$$

where all $I_{2, n}$ are assumed to be functions of $\omega$ and we set, for simplicity, $d_{n, m} \equiv d_{n, m}^{(2)}$. One can easily check that when $\beta=0$ and, accordingly, $d_{n, m}=\delta_{n, m}$ the expression (29) is equivalent to the expression (59) of Ivanov \& Papaloizou (2004).

It is instructive to express natural units in (29). Taking into account that according to our normalisation condition (17) the overlap integral $Q$ can be expressed as $Q=R_{*} M_{*}^{1 / 2} \tilde{Q}$, where $R_{*}$ is the radius of the star and $\tilde{Q}$ is dimensionless. Let us also introduce natural energy and frequency scales associated with the star, $E_{*}=G M_{*}^{2} / R_{*}$ and $\Omega_{*}=\sqrt{G M_{*} / R_{*}^{3}}$, respectively, and use the Press Teukolsky parameter $\eta$ characterising a strength of the tidal interaction,

$$
\eta=\Omega_{*} / \Omega_{o r b}=\frac{1}{\sqrt{1+q}}\left(\frac{R_{m i n}}{R_{*}}\right)^{3 / 2}
$$

With help of these new variables the expression (29) can be rewritten in the form

$$
\Delta E=4\left(\frac{q \tilde{Q}}{(1+q) \eta}\right)^{2} \epsilon E_{*}
$$

\section{Numerical solutions of linear tidal problem for orbital planes inclined to the equatorial plane of the star}

Here we describe numerical solutions for tidal encounters of a planet with a star when the orbit is not coplanar with its equatorial plane. We obtain the energy and $Z$ component of the angular momentum exchanged due to tides acting on the star. This in turn can be related to the rate of orbital circularisation starting from high eccentricity (Papaloizou \& Ivanov 2007).

The numerical procedures follow closely those described in Papaloizou \& Ivanov (2010) that were applied to tidal encounters with a polytrope of index $n=1$ but with appropriate modifications to consider a polytrope with $n=3$. These are described below. Only coreless models are considered here. As in Papaloizou \& Ivanov (2010) grid resolutions of $200 \times 200$ and $400 \times 400$ have been considered. 


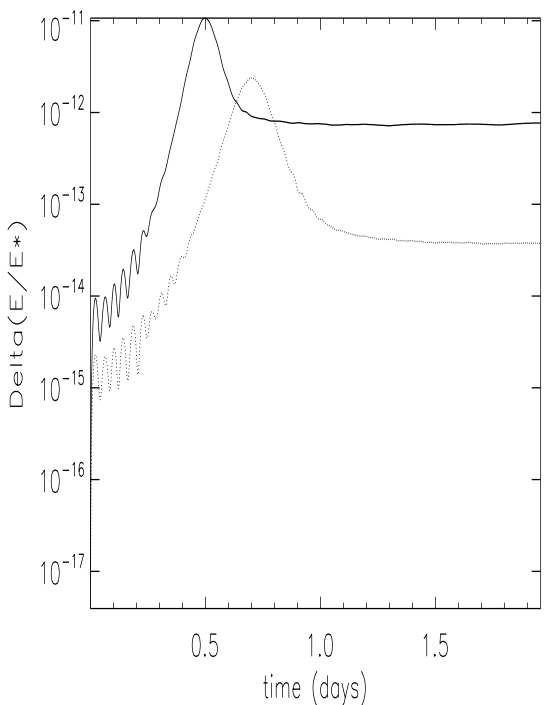

(a)

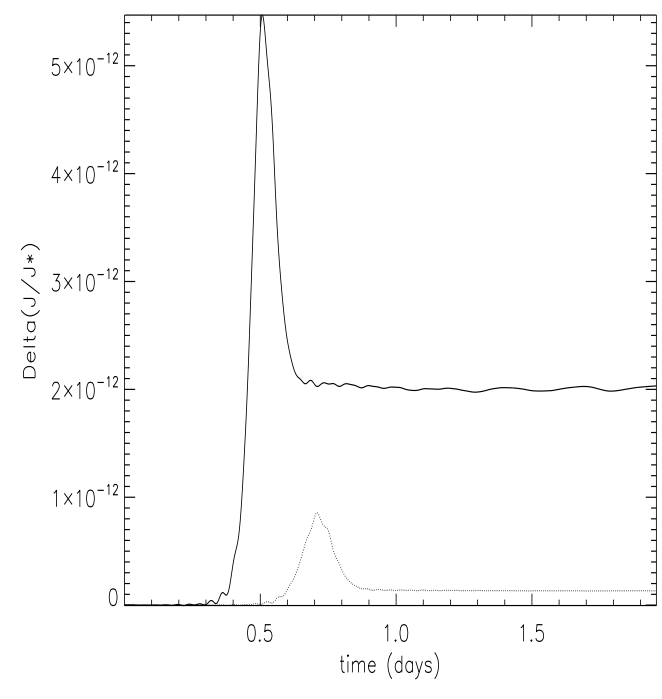

(b)

Fig. 1 a) The energy, in units of $E_{*}$, transferred to the non rotating star as a result of a parabolic encounter with $\eta=4 \sqrt{2}$ (upper solid curve) and $\eta=8$ (lower dotted curve). The mass ratio $q=10^{-3}$.

b) The $z$ component of the angular momentum, in units of $J_{*}=M_{*} \sqrt{G M_{*} R_{*}}$, transferred to the non rotating star for $\eta=4 \sqrt{2}$ (upper solid curve) and $\eta=8$ (lower dotted curve). The mass ratio $q=10^{-3}$.

4.1 A simple stellar model

We adopt a simple model corresponding to a spherically symmetric polytrope of index $n=3$. It is in hydrostatic equilibrium such that

$$
\frac{1}{\rho} \frac{d P}{d r}=\frac{d \psi}{d r}
$$

where $\rho$ is the density, $P=K \rho^{1+1 / n}$ is the pressure, with $K$ being the polytropic constant and $\psi$ is the gravitational potential arising from the stellar matter. This satisfies the Poisson equation

$$
\frac{d}{d r}\left(r^{2} \frac{d \psi}{d r}\right)=-4 \pi G \rho
$$




\subsection{Linearised equations of motion}

We assume that the star rotates with uniform angular velocity $\boldsymbol{\Omega}$ directed parallel to the $Z$ axis. The hydrodynamic equations for adiabatic perturbations induced by an orbiting planet in a frame corotating with the star are

$$
\frac{\partial \mathbf{v}}{\partial t}+2 \boldsymbol{\Omega} \times \mathbf{v}=-\frac{P^{1 / \gamma}}{\rho} \nabla\left(P^{\prime} / P^{1 / \gamma}\right)-\xi_{r} \omega_{B V}^{2} \mathbf{e}_{r}+\nabla U+\nabla \psi^{\prime}+\frac{\mathbf{f}_{\nu}}{\rho},
$$

where the Brunt Vaisala frequency $\omega_{B V}$ is given by

$$
\omega_{B V}^{2}=\frac{1}{\rho} \frac{d P}{d r}\left(\frac{1}{\rho} \frac{d \rho}{d r}-\frac{1}{\gamma P} \frac{d P}{d r}\right),
$$

$\mathbf{v}=\left(v_{r}, v_{\theta}, v_{\phi}\right)$ is the Eulerian velocity perturbation, $P^{\prime}$ is the Eulerian pressure perturbation, $\mathbf{f}_{\nu}$ is the viscous or diffusive force per unit volume, $\psi^{\prime}$ is the perturbation of the gravitational potential due to the star and as indicated above $U$ is the external forcing tidal potential. The adiabatic exponent $\gamma$ is taken to be constant and equal to $5 / 3$. The velocity perturbation $\mathbf{v}$ is related to $\boldsymbol{\xi}=\left(\xi_{r}, \xi_{\theta}, \xi_{\phi}\right)$, the Lagrangian displacement vector, through

$$
\frac{\partial \boldsymbol{\xi}}{\partial t}=\mathbf{v}
$$

The linearised continuity equation gives

$$
\rho^{\prime}=-\nabla \cdot(\rho \boldsymbol{\xi})
$$

where $\rho^{\prime}$ is the density perturbation. This together with the adiabatic condition gives the Eulerian pressure perturbation as

$$
P^{\prime}=-\xi_{r} \frac{d P}{d r}-\gamma P \nabla \cdot(\rho \boldsymbol{\xi})
$$

The gravitational potential perturbation $\psi^{\prime}$ satisfies the linearised Poisson equation

$$
\nabla^{2} \psi^{\prime}=-4 \pi G \rho^{\prime}
$$

Equation (41) may be solved by expanding the right hand side in a series of spherical functions. We simplify the problem by noting that we consider solutions the tidal problem by combining solutions of equation (36) where the angular dependence of $U$ is through a single spherical function. To evaluate $\psi^{\prime}$ we retain only the spherical function associated with the forcing in the decomposition of $\rho^{\prime}$. This is an exact procedure in the non rotating case and should be a reasonable approximation in the rotating case as the potential perturbations associated with high order spherical functions are expected to be small. It amounts to retaining only the quadrupole components of the gravitational potential for the problem on hand, a procedure that has been used successfully in problems of stellar dynamics (eg. Allen et al. 1990). 
Note also hat the centrifugal term is absent in equation (36) being formally incorporated into the potential governing the static equilibrium of the unperturbed star and there is no unperturbed motion in the rotating frame. As in our previous work (eg. Papaloizou \& Pringle 1981, Papaloizou \& Ivanov 2010) we shall neglect centrifugal distortion of the basic equilibrium which enables us to adopt a spherically symmetric unperturbed model and density distribution. We consider prograde encounters, retrograde encounters and encounters for which the orbital plane is at right angles to the stellar equatorial plane.

\subsection{Tidal forcing potential for prograde and retrograde encounters}

For prograde encounters the general expression (3) for the tidal potential reduces to the specific form

$$
U=\mathcal{R}\left[\frac{3 G M}{4 R}\left(\frac{r}{R}\right)^{2} \sin ^{2}(\theta) \exp \left(2 i(\phi-\Phi)-\frac{G M}{4 R} \frac{r^{2}}{R^{2}}\left(3 \cos ^{2} \theta-1\right)\right],\right.
$$

where $\mathcal{R}$ indicates that the real part is to be taken and $R(t)$ and $\Phi(t)$ are given by (8) - (10). The same expression may be used for a retrograde encounter by making the replacement $\Phi \rightarrow-\Phi$.

4.4 Tidal forcing potential for encounters with $\beta=\pi / 2$

In this case when pericentre is in the equatorial plane we may write

$$
\begin{aligned}
U= & \frac{G M}{4 R}\left(\frac{r}{R}\right)^{2} \mathcal{R}\left[-3 \sin ^{2} \theta \cos ^{2} \Phi \exp (2 i \phi)\right. \\
& \left.-6 \mathrm{i} \sin \theta \cos \theta \sin 2 \Phi \exp (i \phi)+\left(3 \cos ^{2} \theta-1\right)\left(3 \sin ^{2} \Phi-1\right)\right]
\end{aligned}
$$

and when it is on the rotation axis we may write

$$
\begin{aligned}
U= & \frac{G M}{4 R}\left(\frac{r}{R}\right)^{2} \mathcal{R}\left[-3 \sin ^{2} \theta \sin ^{2} \Phi \exp (2 i \phi)\right. \\
& \left.+6 \mathrm{i} \sin \theta \cos \theta \sin 2 \Phi \exp (i \phi)+\left(3 \cos ^{2} \theta-1\right)\left(3 \cos ^{2} \Phi-1\right)\right] .
\end{aligned}
$$

We remark that changing the direction of motion along the orbit by the replacement $\Phi \rightarrow-\Phi$ does not change the energy transfer or the change in the $Z$ component of angular momentum. This corresponds to encounters with $\beta=\pi / 2$ and $\beta=3 \pi / 2$ giving the same results for these quantities. The above tidal potentials consist of a sum of terms with different azimuthal mode number $m$. These can be considered independently and the energy and angular momentum transferred as a result of the action of each term evaluated as the canonical energy and angular momentum obtained after the encounter is over (see below). The results can then be added to get the total. As in Papaloizou \& Ivanov (2010) the encounters are started with the perturber at eight times the pericentre distance with $R$ and $\Phi$ determined from (8) - (10). It can be seen that the results are unaffected by applying an arbitrary complex phase shift to each individual forcing term. 


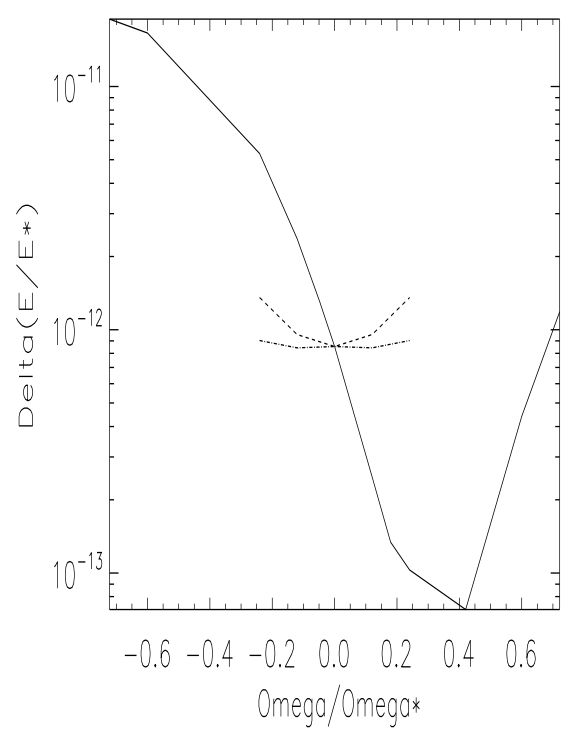

(a)

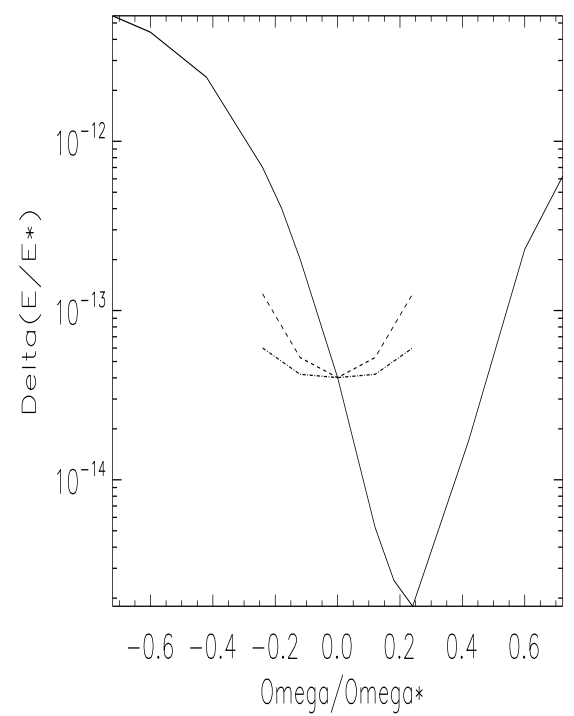

(c)

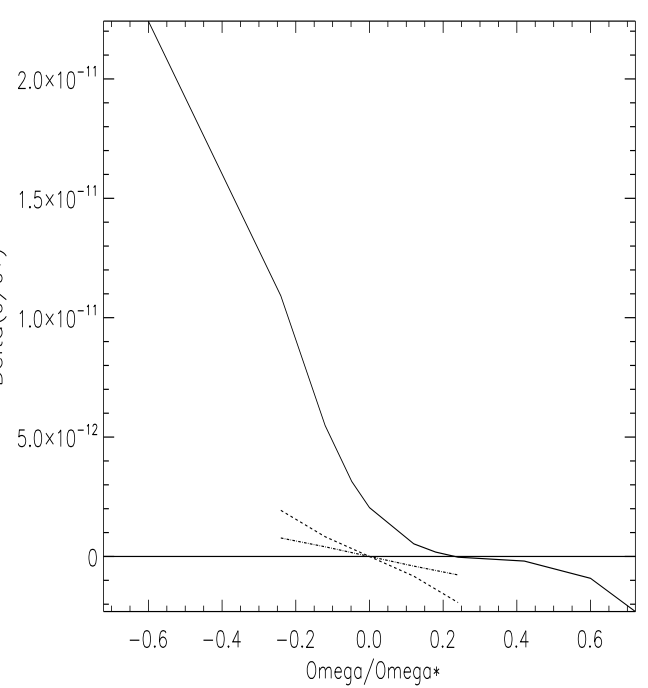

(b)

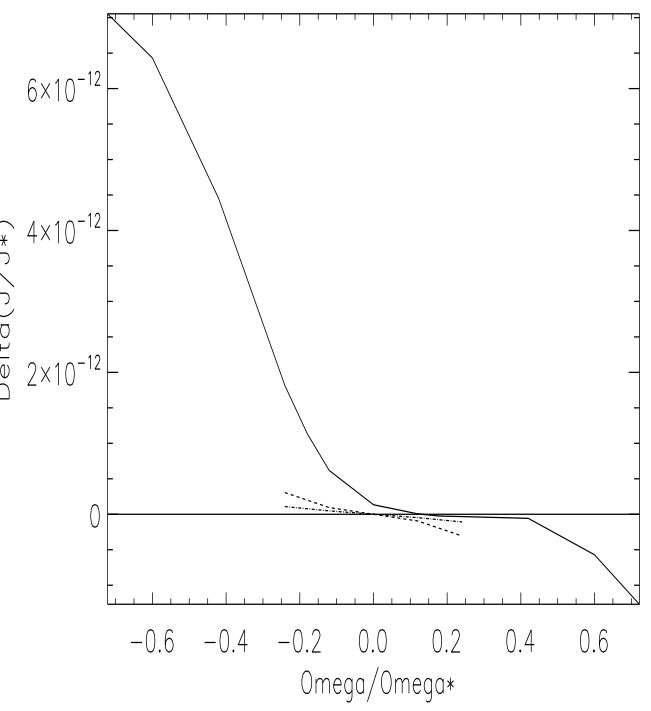

(d)

Fig. 2 a) The energy, in units of $E_{*}$, transferred to the star for $\eta=4 \sqrt{2}$ and $q=$ $10^{-3}$ plotted as a function of $\Omega / \Omega_{*}$. Negative values of the latter correspond to retrograde rotation. The upper dashed curve spanning $(-0.25,0.25)$ shows the energy transferred when the orbital plane is at right angles to the stellar equatorial plane where pericentre is located. The lower dot dashed curve spanning $(-0.25,0.25)$ is for the same parameters except that pericentre is located on the stellar rotation axis. b) The angular momentum, in units of $J_{*}=M_{*} \sqrt{G M_{*} R_{*}}$, transferred to the star. The initially uppermost dashed curve spanning $(-0.25,0.25)$ shows the $z$ component of the angular momentum transferred when the orbital plane is at right angles to the stellar equatorial plane where pericentre being is located. The initially lowermost dot dashed curve spanning $(-0.25,0.25)$ corresponds to the same parameters except that pericentre is located on the stellar rotation axis. c) As in a) but for $\eta=8$. d) As in b) but for $\eta=8$. 
4.5 Addition of diffusive effects

In order to avoid potential numerical problems arising from the excitation the a dense or continuous spectrum of normal modes that may be associated with a rotating star, we follow Papaloizou \& Ivanov (2010) and incorporate a diffusive force per unit mass, $\mathbf{f}_{\nu}$, in the simulations. For models with non zero rotation this is taken to be of the form

$$
\mathbf{f}_{\nu}=\frac{\partial}{\partial r}\left(\rho \nu \frac{\partial \mathbf{v}}{\partial r}\right)+\frac{1}{r^{2}} \frac{\partial}{\partial \mu}\left(\rho \nu \sin \theta \frac{\partial \mathbf{v}}{\partial \mu}\right),
$$

where the diffusion coefficient or effective kinematic viscosity $\nu=\nu_{0} \sqrt{G M_{*} R_{*}}$ was taken to be a constant. For simulations with a grid resolution of $200 \times 200$ we adopted $\nu_{0}=1.3 \times 10^{-6}$ and for a grid resolution of $400 \times 400$ we adopted $\nu_{0}=6.5 \times 10^{-7}$. For non rotating models $\nu_{0}=0$. We remark that energy and angular momentum transfers measured by evaluating the canonical energy and angular momentum just after the encounter has completed are robust to changes in numerical resolution and diffusivity.

\subsubsection{The canonical energy}

When the Lagrangian pressure perturbation vanishes at the outer boundary, the canonical energy (defined in the rotating frame) appropriate to forcing through a Fourier mode with azimuthal mode number, $m$, may be written as

$$
\begin{aligned}
E_{c} & =0.25\left(1+\delta_{m, 0}\right)\left[\int_{V} \rho\left(|\mathbf{v}|^{2}+\omega_{B V}^{2}\left|\xi_{r}\right|^{2}+\left|P^{\prime}\right|^{2} /(\gamma P)\right) d \tau\right. \\
& \left.-\int \frac{\left|\nabla \psi^{\prime}\right|^{2}}{4 \pi G} d \tau-\int_{A}\left|\xi_{r}\right|^{2}(d P / d r) d S\right],
\end{aligned}
$$

where $\left.\delta_{m, 0}\right)$ is the Kronecker $\delta$. The canonical energy contains contributions from the pressure contribution, buoyancy and self-gravity. The last two were not included in Papaloizou \& Ivanov (2010) where a polytrope with $n=1$ was considered using the Cowling approximation. The first volume integral is taken over the volume of the star $V$, while the second giving the gravitational energy is taken over all space. The surface integral is taken over the surface area $A$. The contribution of the surface term formally vanishes when the density at the surface is zero. When the density at the surface is relatively small as in our model, this term gives a negligible contribution. When tidal forcing operates, the time rate of $E_{c}$ gives the rate of energy transfer to the star. After the encounter tidal forcing tails away and $E_{c}$ is conserved in the absence of dissipation. In the presence of dissipation it decays with time.

Let us stress the difference between the energy defined in the rotating frame and in the inertial frame. The energy transfer in the rotating frame $\Delta E_{r}=E_{c}$ is related to the energy transfer in the inertial frame (see equation (33) and the next Section ) as

$$
\Delta E=\Delta E_{r}+\Omega \Delta J_{z},
$$


where $J_{z}$ is the transfer of $z$ th component of angular momentum, which may be equated to the canonical angular momentum, $J_{c}$, contained in the perturbed modes, see below. While it is more convenient to operate with $\Delta E_{r}$ in our numerical work, the analytical work as well as different astrophysical applications are concerned directly with $\Delta E$. Therefore, we illustrate the behaviour of $\Delta E_{r}$ in this Section when discussing our numerical results and $\Delta E$ in the next Section where numerical and analytic results are compared.

\subsubsection{The canonical Angular momentum}

The corresponding canonical angular momentum is given by

$$
J_{c}=-\mathcal{I} m\left[0.5 m \int_{V} \rho\left(\xi_{r}^{*} v_{r}+\xi_{\theta}^{*} v_{\theta}+\xi_{\phi}^{*} v_{\phi}+2 \Omega\left(\xi_{r} \xi_{\phi}^{*} \sin \theta+\xi_{\theta} \xi_{\phi}^{*} \cos \theta\right)\right) d \tau\right],
$$

where $\mathcal{I} m$ indicates that the imaginary part is to be taken. Here we recall that the state variables are complex. This behaves in a manner analogous to the canonical energy, but as applied to the total angular momentum content of the star.

\section{Numerical results}

5.1 The energy and angular momentum transfer for a close encounter

The energy, in units of $E_{*}$, transferred to a non rotating star during parabolic encounters with a planet with mass ratio $q=10^{-3}$ for $\eta=4 \sqrt{2}$ and $\eta=8$ is shown as a function of time in Fig. 1(a). These results are for the $m=2$ tide which is the dominant one (Papaloizou \& Ivanov 2004, 2010). The corresponding angular momentum transferred to the star is plotted as a function of time in Fig. 1(b). The energy and angular momentum transferred level of at constant values after the tidal interaction is essentially complete, the characteristic time associated with the encounter being about 1 day. This corresponds to the excitation of the normal modes associated with the rotating star which in this case correspond to $f, p$ and $g$ modes. It is found that $g$ modes up to order 9 may be significant (see Lee \& Ostriker 1986). In practice we measure transferred quantities at $t=0.75 d$ when $\eta=4 \sqrt{2}$ and at $t=1.4 d$ when $\eta=8$. Our results for the final energies transferred to the star agree with theirs to about the $10 \%$ level.

\subsection{Prograde and retrograde encounters with a rotating star}

The energy, in units of $E_{*}$, transferred to the star as on completion of a parabolic encounter with $\eta=4 \sqrt{2}$ and $q=10^{-3}$ is plotted as a function of $\Omega / \Omega_{*}$ in Fig. 2(a). Negative values of $\Omega / \Omega_{*}$ correspond to retrograde rotation. The angular momentum, in units of $J_{*}=M_{*} \sqrt{G M_{*} R_{*}}$, that is transferred is 


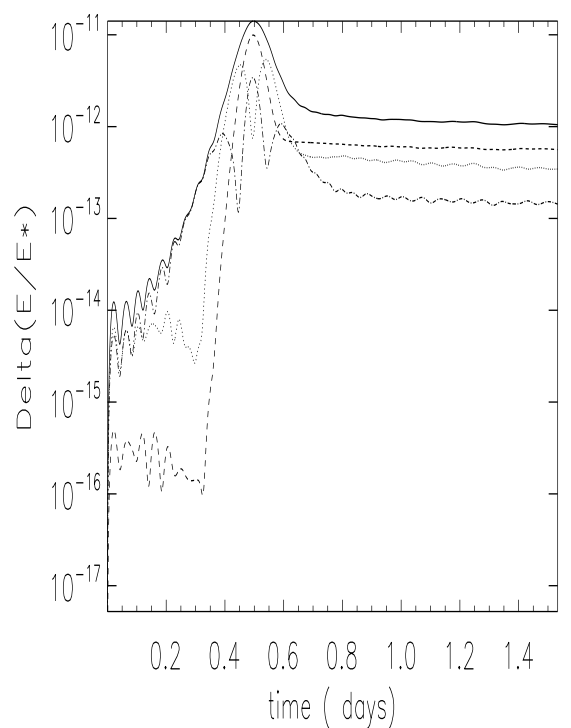

(a)

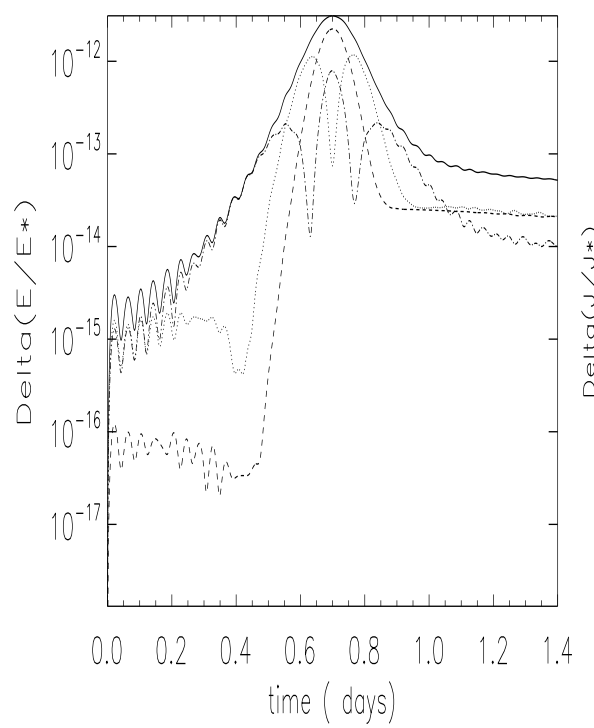

(c)

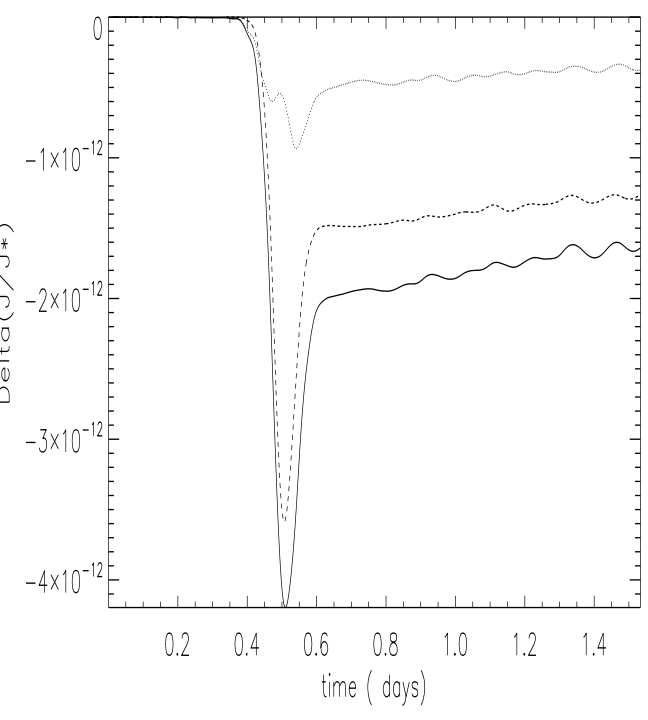

(b)

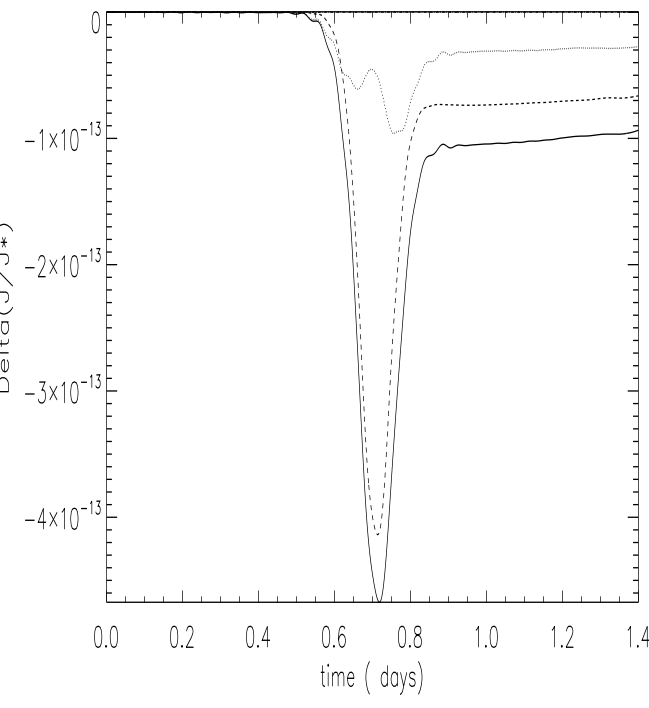

(d)

Fig. 3 a) The energy, in units of $E_{*}$, transferred to the star for $\eta=4 \sqrt{2}, q=10^{-3}$ and $\Omega / \Omega_{*}=0.24$ (uppermost solid curve). The orbital plane was at right angles to the stellar equatorial plane with pericentre located in the latter plane. The initially lowermost dashed curve shows the contribution from terms in the forcing potential with $m=2$. Similarly the initially next lowermost dotted curve corresponds to $m=1$ and the ultimately lowermost dot-dashed curve to $m=0 . \mathrm{b}$ ) The $z$ component of the angular momentum, in units of $J_{*}$, transferred to the star (lowermost solid curve). The ultimately intermediate dashed curve shows the contribution from terms in the forcing potential with $m=2$. The ultimately uppermost dotted curve corresponds to $m=1$. c) As in b) but for $\eta=8$ and $\Omega / \Omega_{*}=0.12$. d) As in c) but for $\eta=8$ and $\Omega / \Omega_{*}=0.12$. 
plotted as a function of $\Omega / \Omega_{*}$ in Fig. 2(b) In Fig. 2(c) and 2(d) the corresponding plots are given for $\eta=8$. The energy transferred decreases as $\Omega$ is increased from zero until a minimum value is attained, after which it increases with $\Omega$ again. On the other hand the energy transferred increases with increasing retrograde rotation attaining values up to more than three orders of magnitude larger than the minimum one for $\Omega / \Omega_{*} \sim-0.6$. when $\eta=8$. Thus circularisation from high eccentricity as a result of stellar tides can be expected to be much more effective for significant retrograde rotation. For $\eta=4 \sqrt{2}$ the minimum energy transfer occurs for $\Omega / \Omega_{*} \sim 0.4$ while for $\eta=8$ this occurs for $\Omega / \Omega_{*} \sim 0.25$. The actual angular velocity at pericentre $\Omega_{p} / \Omega_{*}=\sqrt{2} / \eta$, so that $\Omega_{p} / \Omega_{*}=0.25$ and 0.18 for these two cases respectively. Accordingly this minimum transfer occurs when $\Omega \sim 1.5 \Omega_{p}$, which is approximately the angular velocity for which the angular momentum transfer reverses sign, this transition occurring when $\Omega \sim \Omega_{p}$, (see Figs. 2(b) and 2(d)). This characteristic behaviour was also noted in the case of a polytrope with index $n=1$ and a barotropic equation of state ( Ivanov \& Papaloizou 2004, 2007).

We have also considered encounters for which the orbital plane and the stellar equatorial plane are perpendicular. Cases for which pericentre was in the equatorial plane and on the rotation axis have been considered. The energy transferred to the star for some of these cases is plotted in Figs. 2(a) and 2(c) The component of angular momentum in the $z$ direction transferred to the star is plotted in Figs. 2(b) and 2(d). The energy transferred in these cases is also seen to significantly exceed the minimum that is transferred in the prograde case.

To look at such encounters more closely, the energy, in units of $E_{*}$, transferred to the star as a result of a parabolic encounter with $\eta=4 \sqrt{2}$ and $q=10^{-3}$ for $\Omega / \Omega_{*}=0.24$ when the orbital plane was at right angles to the stellar equatorial plane where pericentre was located is plotted as a function of time in Fig. 3(a), The $z$ component of angular momentum transferred is plotted in Fig. 3(b), The contributions from the terms in the forcing potential with $m=2, m=1$ and $m=0$ are indicated. Clearly the last of these does not affect the $z$ component of angular momentum. Note that significant contributions come from $m=1$ as well as $m=2$. The corresponding results for $\eta=8$ and $\Omega / \Omega_{*}=0.12$ are shown in Figs. $3(\mathrm{c})$ and $3(\mathrm{~d})$.

\section{Comparison of numerical and analytic results and circularisation time scales}

\subsection{Numerical versus analytic results}

In order to calculate the analytical value of the energy transfer, $\Delta E$, we should know the values of the mode eigen frequencies, the overlap integrals and the frequency splitting coefficients $\beta_{r}$. We take into account the $f$-mode, 5 low order $p$-modes and $14 \mathrm{~g}$-modes, which give the most important contribution to the energy transfer. The eigen frequencies and the overlap integral are taken 


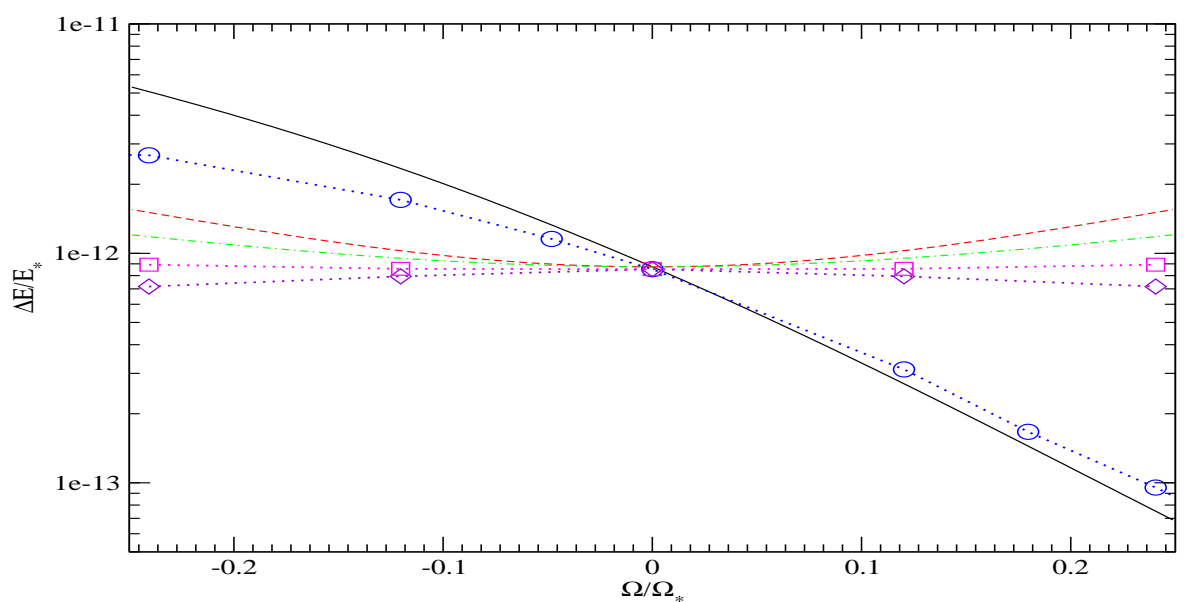

a)

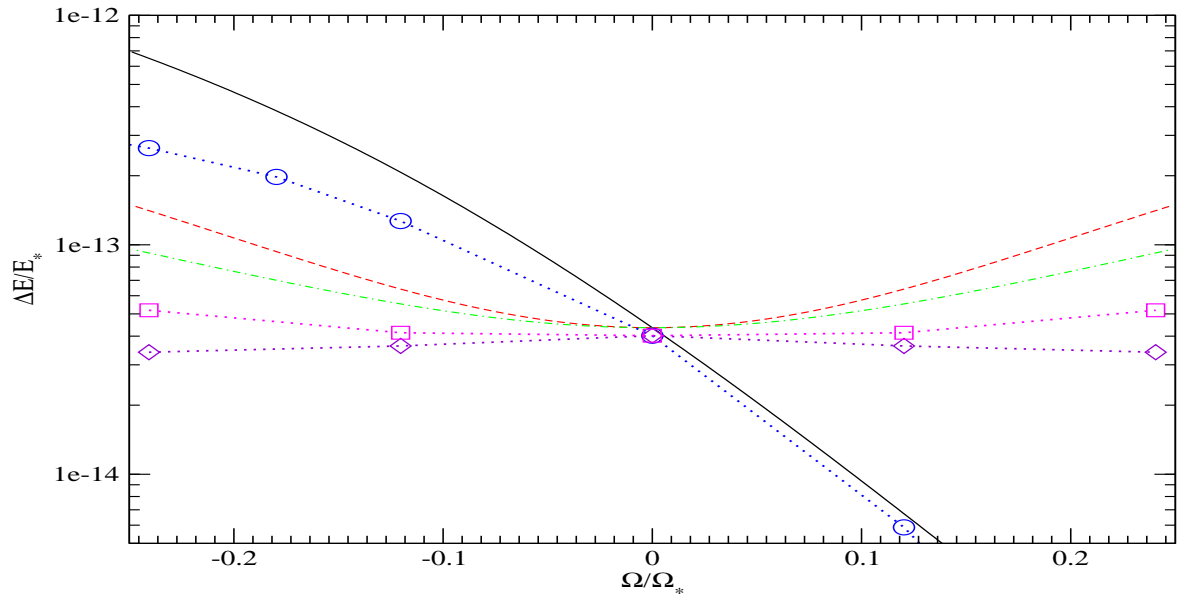

b)

Fig. 4 a) Analytic and numerical dependencies of the energy transferred to the star in units of the characteristic energy $E_{*}\left(E_{*}=3.8 \cdot 10^{48}\right.$ ergs for a star of solar mass and radius) on the angular velocity of the star in units of the characteristic frequency $\Omega_{*}\left(\Omega_{*}=6.23 \cdot 10^{-4} s^{-1}\right.$ for a star of solar mass and radius), for $\eta=4 \sqrt{2}$. See the text for a description of the different curves and symbols. b) Same as a) but for $\eta=8$. 
from the papers Lee \& Ostriker (1986) and Ray et al (1987). The coefficients $\beta_{r}$ for the $f, p$ and several low order $g$-modes are taken from the paper Saio (1981). For the rest of $g$-modes we use the WKBJ results that $\beta_{r} \approx 1-\frac{1}{L(L+1)}$, where $L=2$ in our case, see e.g. Christensen-Dalsgaard (1998).

In Figs. 4(a) and 4(b) we show the dependencies of the energy transferred to the star as a result of the planet flyby on the angular velocity of the star, for $\eta=4 \sqrt{2}$ and $\eta=8$, respectively. The solid, dashed and dot dashed curves represent the analytic results while the symbols indicate the corresponding numerical results. The solid curve and circles correspond to the situation when the equatorial and orbital planes coincide $(\beta=0)$. The dashed curve and squares correspond to an orbit with normal to the orbital plane perpendicular to the rotational axis and the direction to pericentre laying in the equatorial plane $(\beta=\pi / 2$ and $\gamma=\pi / 2)$. The dot dashed curve and diamonds are calculated for the same $\beta=\pi / 2$ but for the direction to pericentre being aligned with the rotational axis, and, accordingly, $\gamma=\pi$. Note the one can either consider negative values of $\Omega$ as in Figs. 3(a) $[3(\mathrm{~d})$, or a change of the inclination angle such that $\beta \rightarrow \beta+\pi$ keeping the value of $\Omega$ positive. Both approaches are physically equivalent and give the same results. In what follows we describe the results corresponding to positive (negative ) values of $\Omega$ as prograde (retrograde ) and the results corresponding to $\beta=0(\beta=\pi / 2)$ as planar (perpendicular). As seen from Figs. 4(a) and 4(b) in the planar case the analytic and numerical results corresponding to positive rotational frequencies are in good agreement, while the results corresponding to negative frequencies deviate, leading to systematically larger analytic values of $\Delta E$ as compared to the numerical determined ones. The deviation is of the order or smaller than $50 \%$ for $-\Omega / \Omega_{*}<0.1$. For the largest value of $-\Omega / \Omega_{*} \approx 0.25$ shown, it is about a factor of two for $\eta=4 \sqrt{2}$ and about a factor of 2.5 for $\eta=4 \sqrt{2}$. In the perpendicular cases both analytic and numerical results show much weaker dependence on $\Omega$. The analytic curves again correspond to larger values of $\Delta E$, with results deviating most significantly from the numerical ones at large values of $|\Omega|$. The degree of deviation behaves in a similar manner to the planar case. Note that the analytic curves always grow with increasing $|\Omega|$ while the numerical curve corresponding to $\gamma=\pi / 2(\gamma=0)$ is weakly increasing (decreasing ) with $\mid \Omega$.| This weak growth (decrease) is probably within numerical errors and should be treated with caution.

The origin of the deviation at large values of $-\Omega / \Omega_{*}$ is unclear at present. It cannot be fully accounted for by the increasing importance of non-linear corrections to the rotational shift of eigen frequencies given by equation (19) with increasing $|\Omega|$. We found that phenomenological non-linear corrections provided by Lai (1997) do not significantly affect our analytic results for the range of $\Omega$ considered. However, for the values of $\eta$ considered, the most significant contributions to the energy transfer are provided by g-modes of order of $9-10$. The eigenfrequencies of these modes being $\sim 0.5 \Omega_{*}$ are a factor of

4 Let us recall that here we show the energy defined in the inertial frame, which is related to the energy defined in the rotating frame discussed in Section 5 through equation (47). 
$\sim 2.5$ larger than the maximum angular velocity considered. Thus they are approaching the so called inertial regime for which eigenfrequencies are less than $|2 \Omega|$ in magnitude. It is possible that the overlap integrals become significantly modified in such a situation. This issue will be explored in future work.

6.2 The time scale for the initial stage of tidal circularisation

\subsubsection{A basic approach}

In principle, the results reported above can be used in numerical experiments in which a system of gravitationally interacting planets is considered, it being assumed that once a planet has obtained a sufficiently small value of its orbital angular momentum as a result of planet-planet gravitational scattering, it can lose orbital energy due to tidal interaction with the central star during successive periastron passages. If the effect of tidal interaction on changing the orbital energy becomes much larger than residual effects associated with planet-planet scattering, the planet is said to be tidally captured by the star. After being tidally captured, the planet interacts mainly with the central star due to dynamic and quasi-static tides exerted in itself and in the star. Under certain conditions discussed in the next section the orbital energy decreases, either systematically or on average, while the orbital period and orbital eccentricity also decrease. In this way it is possible to account for at least some of the so-called "Hot Jupiters" - giant planets with orbital periods ranging from a fraction of a day to several days.

The central star can rotate, with its rotation axis inclined with respect to the normal to the orbital plane by an angle $\beta$, after the tidal capture. This angle is determined by the particular sequence of planet-planet interactions that led to the tidal capture. Since retrograde tidal interactions that transfer energy to the star are stronger than prograde ones, while the angle $\beta$ may be random or have a weak correlation with the direction of stellar rotation, this property of the tidal interaction may influence the observed relative number of Hot Jupiters on retrograde and prograde orbits. Thus it is important to estimate the potential magnitude of this tidal effect.

As Hot Jupiters may have been formed when the star was young, in making such an estimate it is very important to account for the fact that the angular velocity of the star, $\Omega$, is a function of stellar age. This function can be a rather complicated and is expected to be different for stars with different masses and formation histories (e.g. Scholz 2009). In this paper we make only preliminary qualitative estimates of the importance of tides exerted in a rotating star on the formation of Hot Jupiters. Accordingly, we use the simplest plausible form of this function known as the Skumanich law (Skumanich 1972), which states that the angular velocity is inversely proportional to square root of the stellar 
age. Thus we adopt the following expression for the stellar angular velocity:

$$
\Omega=2.4 \cdot 10^{-6} \sqrt{\frac{5 \cdot 10^{9} y r}{t}} s^{-1} .
$$

Here $\Omega$ is normalised so that the star has a rotation period of one month at an age of $t=5 \cdot 10^{9} \mathrm{yr}$.

In this paper we do not consider the planet-planet interaction stage, leaving this for a future work. Here we assume that tidal capture has happened and the planet interacts only with the star. It supposed to be initially on a highly eccentric ( essentially parabolic) 5 orbit with well defined values of the angles $\beta$ and $\gamma$ and have a value of the orbital angular momentum corresponding to a given value of the parameter $\eta$ defined in equation (32). The orbital energy, eccentricity and period are gradually changing their values due to tidal interactions on some characteristic evolution time scale, $t_{e v}$, specified below. As discussed in e.g. Ivanov \& Papaloizou (2004,2007), orbital evolution as a result of tides acting in the planet approximately conserve orbital angular momentum because the planet spins up (or slows down) by tides to a state of rotation corresponding to the so-called pseudo-synchronisation, where the angular momentum exchange between the orbit and pulsational modes of the planet is absent. This happens on a quite short time scale due to a small moment of inertia of the planet compared to that of the orbit.

In the case of tides exerted in the star we have an opposite situation where the stellar moment of inertia is typically much larger than the orbital one. Therefore, the star cannot be brought by action of tides to the state of pseudosynchronisation during the evolution of the system, and some amount of angular momentum is transferred from the orbit to the star in the course of it thus decreasing the value of the orbital angular momentum. We would like to estimate this value assuming that tides are effective all the way down to smallest values of the orbital eccentricity. Let us stress again that the dynamic tides discussed in this paper are effective only at sufficiently large values of the eccentricity $e \sim 1$ ( see the next section). However, it seems plausible to suppose that processes not considered here, such as the nonlinear breaking of excited waves, critical latitude phenomena in the planet, or friction, resulting from for example turbulence, acting on quasi-static tides could be important when the eccentricity is moderate and small, and that they can eventually bring the system to the state having a low eccentricity $e \sim 0$ typical for the Hot Jupiters (e.g. Barker \& Ogilvie 2009).

It is well known that the mode energy, $E_{m}$, is related to the mode angular momentum, $J_{m}$, as $E_{m}=\omega_{m} J_{m} / m$, see e.g. Friedman \& Schutz (1977). Accordingly, the total amount of energy and angular momentum, $E_{t}$ and $J_{t}$,

5 A planet with orbital parameters typical for the stage of tidal interaction right after the tidal capture would have its periastron distance of order of several (say, 4) stellar radii, while its semimajor distance would be of order of a few (say, 10) astronomical units (e.g. Nagasawa et al 2008). For such parameters orbital eccentricity $e$ is of order of 0.998 and approximation of tidal encounters as happening on parabolic flybys is well justified. 
transferred from the orbit to the star obeys a similar relation, where, in general, we should sum over all modes of the star. In our case of a slowly rotating star only $m=2 g$-modes of sufficiently high order give a significant contribution to the sum. For our problem the corresponding eigenfrequencies of these modes can be roughly estimated as $\omega_{n} \approx 0.5 \Omega_{*}$, and we obtain $E_{t} \approx \Omega_{*} J_{t} / 4$. After the tidal circularisation has happened assuming that the tides induced in the star dominate over those in the planet we can equate the total mode energy $E_{t}$ to the orbital binding energy of the planet after the circularisation, $E_{f}=G M M_{*} /\left(2 a_{f}\right)$, where $a_{f}$ is the final value of the orbital semi-major axis, and we suppose that $e \sim 0$, and, accordingly, the orbital angular momentum $J_{f}=M \sqrt{G M_{*} a_{f}}$. From these expressions we obtain

$$
\frac{J_{t}}{J_{f}} \approx 2\left(\frac{R_{*}}{a_{f}}\right)^{3 / 2}=\frac{1}{\sqrt{2} \eta},
$$

where in the last equality we use equation (32) setting $q=0$ there, and assuming that $J_{t} / J_{f}$ is small, and, therefore, the orbital angular momentum is approximately conserved during the tidal circularisation. In this case we can set $R_{\min }=a_{f} / 2$ in (32). As follows from equation (51) below, $J_{t} / J_{f}<0.1$ for the Hot Jupiters with the orbital periods $>2.4$ days. As seen from Figs. 5(a) and $5(\mathrm{~b})$ the stellar tides can dominate for even larger periods, accordingly the assumption of approximate conservation of the orbital angular momentum can then be justified 6 .

This fact also allows us to link a value of $\eta$ to a value of orbital period after the tidal circularisation process has been completed and the planet's orbit has became almost circular, $P_{o b s}=2 \pi \sqrt{a_{f}^{3} /\left(G M_{*}\right)}$, as

$$
\eta \approx 3\left(\frac{M_{*}}{M_{\odot}}\right)^{1 / 2}\left(\frac{R_{\odot}}{R_{*}}\right)^{3 / 2} P_{1}
$$

where $P_{1}=P_{o b s} / 1 d a y$. This equation relates an observable quantity, $P_{o b s}$, to the quantity determining the strength of the tidal interactions.

For simplicity, it is assumed below in this section that we can simply add the values of energy transfer during successive periastron passages either due to sufficiently efficient dissipation of energy stored in the modes between periastron passages (e.g. Kumar \& Goodman 1996) or due to the operation of stochastic instability in the system, e.g. Kochanek (1992), Kosovichev \& Novikov (1992), Mardling (1995)a,b, Ivanov \& Papaloizou (2004), (2007). Note that in the latter case the characteristic time scales for orbital circularisation must be understood in some average sense. Some estimates relevant to a more realistic situation are discussed in the next section.

Under this assumption Ivanov \& Papaloizou (2004), (2007) derived an equation determining the rate of change of the orbital semimajor axis, $a$, in the form

\footnotetext{
${ }^{6}$ When the planetary tides dominate, equation (50) clearly overestimates $J_{t} / J_{f}$.
} 


$$
\frac{\dot{a}_{10}}{a_{10}}=-\frac{1}{t_{10}(t) \sqrt{a_{10}}},
$$

where $a_{10}=a /(10 a u)$ and

$$
t_{10}=7 \cdot 10^{8}\left(\sqrt{\frac{M_{*}}{M_{\odot}}} \frac{M_{J}}{M}\right)\left(\frac{R_{p l}}{R_{J}}\right)\left(\frac{10^{-9}}{\epsilon_{D T}}\right) y r,
$$

where $M_{J}, R_{J}$ are the Jupiter mass and radius, respectively, $R_{p l}$ is the planet's radius, and

$$
\epsilon_{D T}=\left(\Delta E+\Delta E_{p l}\right)\left(\frac{R_{p l}}{G M^{2}}\right),
$$

where $\Delta E$ is given by equation (33) and $\Delta E_{p l}$ is the energy transfer to the modes exited in the planet 7 . This is calculated in Ivanov \& Papaloizou (2004),( 2007 ) for the value of $\eta$ given by (51) corresponding to a specified final orbital period. Note that both $\Delta E$ and $\Delta E_{p l}$ depend on time. In the case of $\Delta E$ this is through the dependence of the stellar angular velocity on time, assumed to be given by equation (49). In the case of $\Delta E_{p l}$ this is mainly through the evolution of the planet's radius on time. Following Ivanov \& Papaloizou ( 2004), (2007) we assume in this paper that this evolution is the same as the evolution of an isolated planet in which the planet's radius shrinks with time. Thus, we neglect the possible influence of tidal heating on the planet's structure.

Equation (54) can be integrated to give

$$
a_{10}(t)=a_{i n}\left(1-\frac{1}{2 \sqrt{a}_{i n}} \int_{t_{i n}}^{t} \frac{d t^{\prime}}{t_{10}\left(t^{\prime}\right)}\right)^{2},
$$

where $a_{i n}\left(t_{i n}\right)$ is the 'initial' value of the planet's semimajor axis (in units of $10 a u)$ just after tidal capture has occurred. Let us define the evolution time scale as the time corresponding to $a_{10}$ being formally equal to zero. From (55) we get

$$
\int_{t_{i n}}^{t_{e v}} \frac{d t^{\prime}}{t_{10}\left(t^{\prime}\right)}=2 \sqrt{a}_{i n}
$$

which provides an implicit equation for determination of $t_{e v}$. However, let us recall that the evolution time scale so defined describes the evolution of the semi-major axis at large scales, of the order of $1-10 a u$ or larger, under the assumption that the dynamical tides can effectively transfer the orbital energy to energy associated with normal modes and thermal energy of the star and the planet. At smaller scales the dynamic tides are clearly ineffective and other processes (e.g.due to so called quasi-static tides ) must be considered to explain final stages of circularisation of exoplanets, see the next section.

\footnotetext{
7 Note that in Ivanov \& Papaloizou (2004), (2007) there is a misprint in their equations defining $t_{10}$. Namely, the value of $t_{10}$ is proportional to $\frac{M_{*}}{M_{\odot}}$ there instead of the correct square root dependence of this ratio as in (53). However, in these papers only the case $M_{*}=M_{\odot}$ was considered and this misprint has no influence on the results of the papers.
} 
The results of the solution of this equation for $a_{i n}\left(t_{i n}\right)=1$ corresponding to an initial orbit with semi-major axis of $10 \mathrm{au}$ and a central solar mass are shown in Figs 5(a), 5(b), for $M=1 M_{J}$ and $M=5 M_{J}$, respectively. In both cases it is assumed that the planet is in a state of rotation corresponding to so-called pseudosynchronization, see Ivanov \& Papaloizou (2004), (2007). We consider only the case

of the planet's orbit being coplanar with the stellar equatorial plane but with both retrograde and prograde directions of orbital motion. As seen from Fig. 5(a) when tides are exerted both in the star and in the planet, there is a difference in the circularisation time scale corresponding to the case of retrograde motion as compared to the cases of either a non-rotating star or of prograde motion. Since criteria for the dissipation of the energy in normal modes as well as conditions determining onset of the stochastic instability may be different for the planet and for the star and it may happen that only stellar tides are important for the circularisation problem under certain circumstances, we also plot the curves calculated assuming that $\Delta E_{p l}=0$. As seen from Fig. 5(a) the stellar tides themselves can provide a tidal circularisation time scale smaller than a typical life time of a planetary system of $\sim 5 \cdot 10^{9} \mathrm{yr}$ for $P_{\text {orb }}<4$ days. In Fig. 5(c) we show the ratios of the evolution time scale corresponding to the prograde and retrograde cases to the case of a non-rotating star, for a planet with $M=1 M_{J}$. One can see from this figure that assuming that tides in the star and in the planet are both effective, there is practically no difference between the prograde and non-rotating cases, while the retrograde case has an evolution time scale $1.2-2$ smaller, for final orbital periods in the range 3.5 to 4.5 days. When only the stellar tides are taken into account, the difference between the prograde, retrograde and non-rotating cases can be quite significant for smaller final orbital periods. Since $\Delta E$ grows with the mass of the planet the contribution of stellar tides is more important for the $M=5 M_{J}$ case shown in Fig. $5(\mathrm{~b})$. In this case $t_{e v}$ is practically determined by these tides for $P_{\text {orb }}$ larger than 2-2.5 days and the difference between the retrograde, prograde and non-rotating case is quite prominent.

As mentioned above there is a difference between the numerically and analytically obtained values of $\Delta E$, which is more significant for a sufficiently rapidly rotating star and retrograde orbits. Let us estimate the possible difference in the evolution time scale associated with this assuming that $t_{e v}>10^{7} \mathrm{yr}$. From equation (49) it follows that $\Omega\left(t>10^{7} y r\right)<0.08 \Omega_{*}$, and from Figs. $5(\mathrm{a})$ and $5(\mathrm{~b})$ it follows that when $\Omega / \Omega_{*}<0.08$ the ratio of analytically obtained values of $\Delta E$ to the numerical ones is of the order of or smaller than 1.5. In order to see the effect of tides exerted in the star being reduced by a factor 1.5, we plot the additional dot dot dashed curve in Fig. 5(a) corresponding to the retrograde case with the energy transfer given by equation (33) reduced by a factor of 1.5. It is seen that the curve is very close to the short dashed curve calculated with help of (33). Therefore, it seems that this issue is not significant for a one Jupiter mass planet. It is even less important for more massive planets whose evolution time scales show a stronger dependence on 


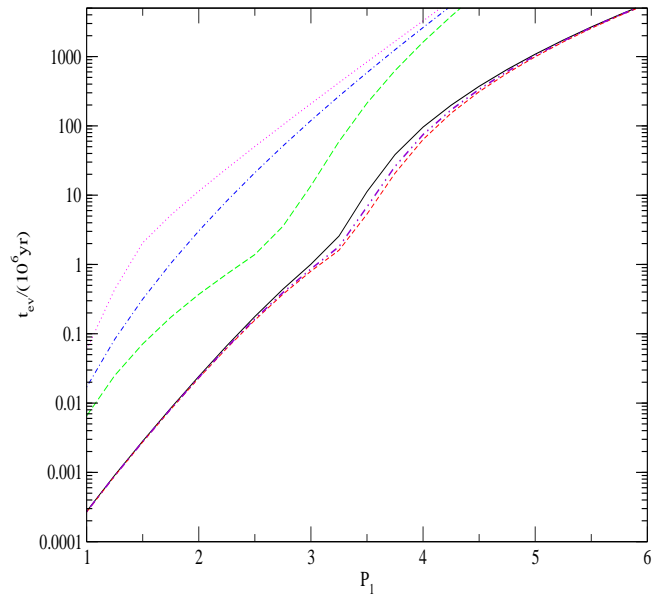

a)

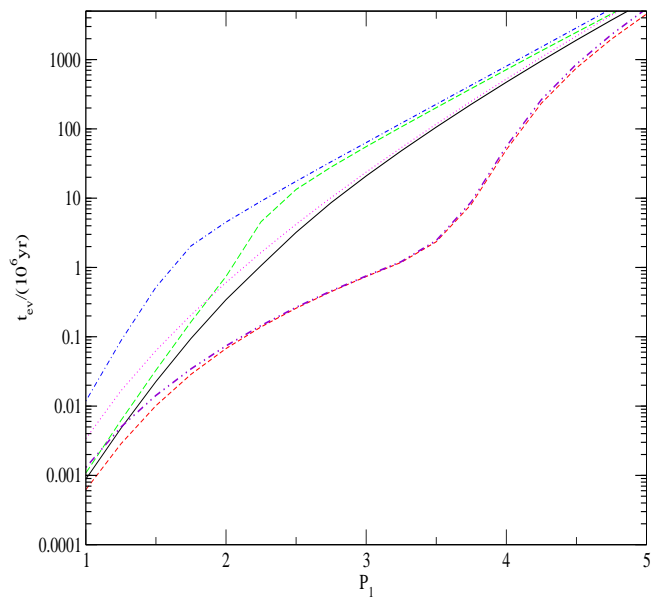

b)

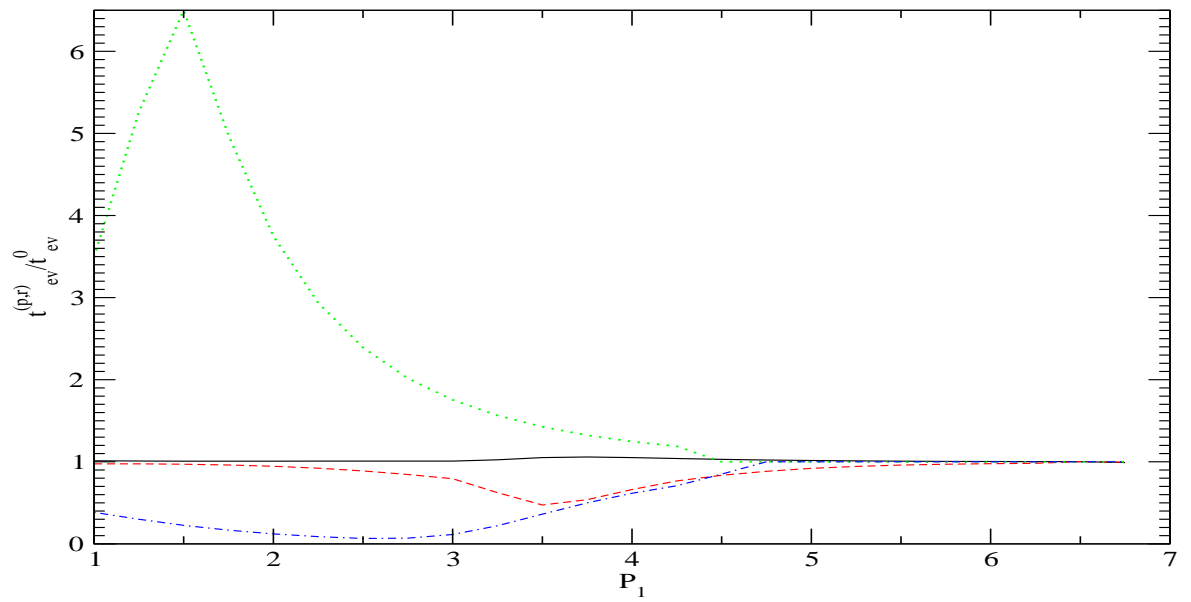

c)

Fig. 5 a) The evolution time scale $t_{e v}$ as a function of $P_{1}$ in units of 1 day. The planet's mass $M=1 M_{J}$. Curves are numbered below in order of increasing magnitude at a fixed value of $P_{1}$ for $P_{1}>2$. The solid curve (3) corresponds to $t_{e v}$ obtained with help of (56) together with $\Delta E$ calculated for a non-rotating star. The short dashed curve (1) is for the retrograde case with $\Omega(t)$ given by 49 . The corresponding plot for the prograde case almost coincides with the solid curve, and so is not shown. The long dashed (4), dot dashed (5) and dotted curves $(6)$ are for tides excited in the star $\left(\Delta E_{p l}=0\right)$. They are for the retrograde, a non-rotating and prograde cases, respectively. The dot dot dashed curve (2) is for the retrograde case with all tides, but with the energy transfer $\Delta E$ reduced by a factor of 1.5 as compared to the value given by (33). b) As in a) but for $M=5 M_{J}$ and with the difference that in this case the long dashed (5), dotted (4) and dot dashed curves (6) determined from tides exerted in the star alone correspond to the retrograde, non-rotating and prograde cases respectively. c) The ratios of the evolution time scales corresponding to the prograde and retrograde cases, $t_{e v}^{p}$ and $t_{e v}^{r}$, to the time scale corresponding to the non-rotating star, $t_{e v}^{0}$, are plotted as functions of $P_{1}$. The solid and dashed curves respectively apply when tides exerted in the star and planet are both included. The dotted and dot dashed curves respectively apply when only tides in the star are included. 
the relative direction of the orbital motion and of the stellar rotation, see e.g. Fig. $5(\mathrm{~b})$

6.2.2 Conditions leading to stochastic instability or effective dissipation of the mode energy

As discussed in Kochanek (1992), Kosovichev \& Novikov (1992), Mardling (1995)a,b, Mardling \& Aarseth 2001, Ivanov \& Papaloizou (2004), (2007), when the orbital semi-major axis is larger than some critical value, $a_{s t}$, stellar and planetary perturbations excited at successive periastron passages have approximately uncorrelated phases. This happens when the phase change of perturbed quantities associated with a normal mode occurring during one orbital period, that arises on account of the change induced in the orbital period itself, is larger than some critical number of order of unity. This change in the orbital period results from the energy interchange between the orbit and the normal modes. This number was estimated numerically by Ivanov \& Papaloizou (2004) in the simplest case of only one mode interacting with the orbital motion due to tides.

In the situation where the phases are uncorrelated the mode energy, which is proportional to square of the mode amplitude grows on average in proportion to the number of periastron passages. This effect is referred to as the stochastic instability.

From the results obtained in Ivanov \& Papaloizou (2004) it follows that the stochastic instability sets in when

$$
a>a_{s t}=\left(\tilde{\omega} \epsilon_{D T}\right)^{-2 / 5}\left(\frac{M_{J}}{M}\right)^{3 / 5}\left(\frac{R_{p l}}{R_{J}}\right) a u,
$$

where $\tilde{\omega}=\omega / \Omega_{*}, R_{p l}$ is the planet's radius and $R_{J}$ and $M_{J}$ are the radius and mass of Jupiter, respectively. We use equation (57) to estimate $a_{s t}$, for definiteness setting $\tilde{\omega}=0.5$ there, which is appropriate for $g$ modes excited in the star and $m_{p l}=M_{J}, R_{p l}=R_{J}$. Since both stellar and planetary tides depend on time $t$ we plot the dependence of $a_{s t}$ on $t$ in Fig. 6(a) for different values of $P_{1}=1,2,3,4$ and 5 .

One can see from this figure that the critical semi-major axis $a_{s t}$ is typically of the order 0.1-10au. Note, however, that the analysis of Ivanov \& Papaloizou 2004 can somewhat overestimate the value of $a_{s t}$ since it was done under the assumption that only one normal mode plays a significant role. For the problem on hand, several modes are excited in the star and and in the planet which could give a comparable contribution to the energy exchanged with the orbit. This is likely to increase 'the degree of stochasticity' in the system leading to smaller values of $a_{s t}$. However, in any case it seems highly improbable that dynamic tides can operate in the stochastic regime at scales much smaller than, say, $0.1 a u$, for the whole of the interesting range of $P_{1}$. Thus, provided that dissipation of mode energy is not unexpectedly large, dynamical tides are not efficient at such scales so that quasi-static tides need be invoked to explain further tidal circularisation. 


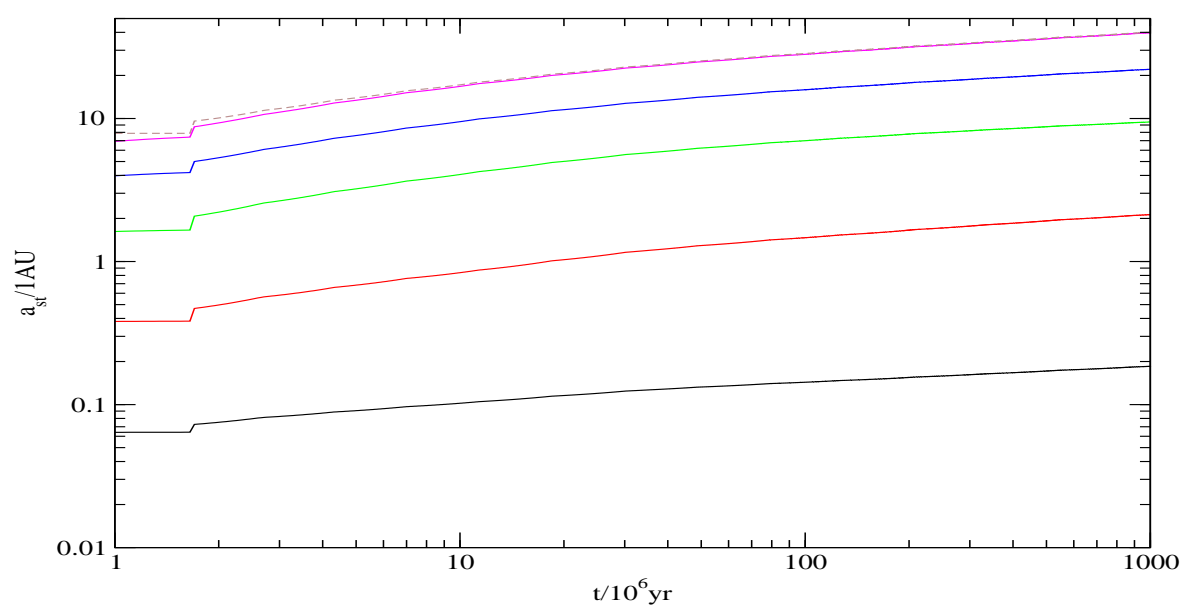

a)

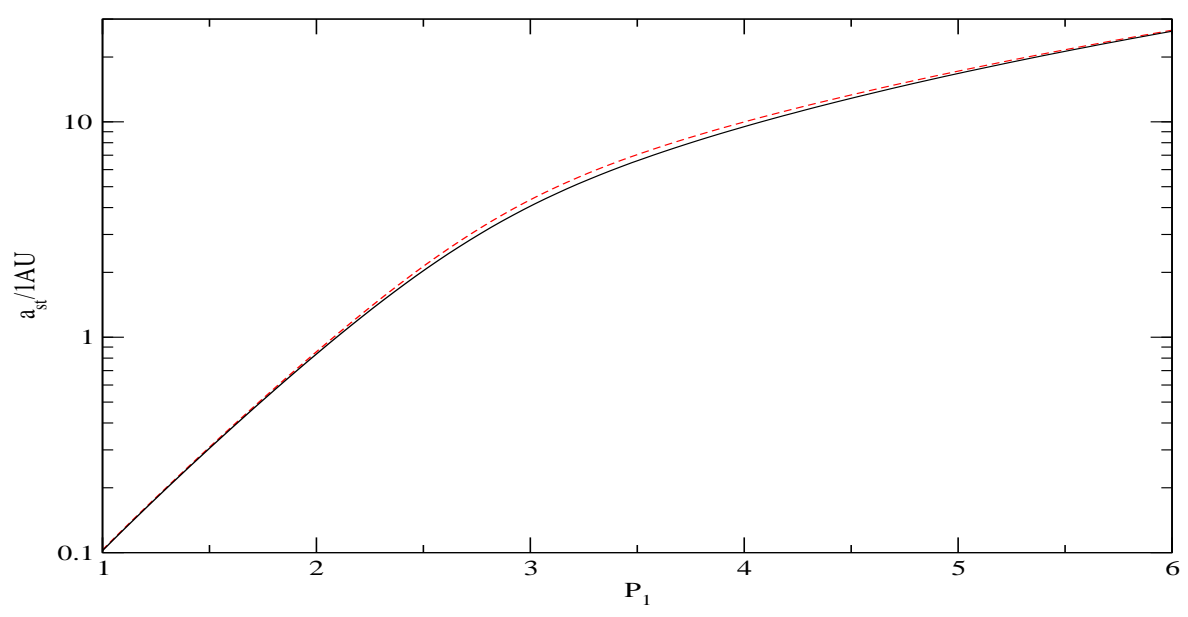

b)

Fig. 6 a) The scale $a_{s t}$ as a function of time $t$. The rotational history of the star is given by (49). Solid curves correspond to the retrograde orbits with values of $P_{1}=1,2,3,4,5$ with curves having larger values at given moment of time corresponding to larger values of $P_{1}$. The difference between the prograde and retrograde orbits is not well pronounced. As an example the dashed curve shows the dependence of $a_{s t}$ on $t$ for the prograde orbit with $P_{1}=5$. b) Same as the upper panel but $a_{s t}$ is shown as a function of $P_{1}$ for a time or stellar age of $t=10^{7} \mathrm{yr}$. The solid and dashed curves show the retrograde and prograde cases, respectively. 


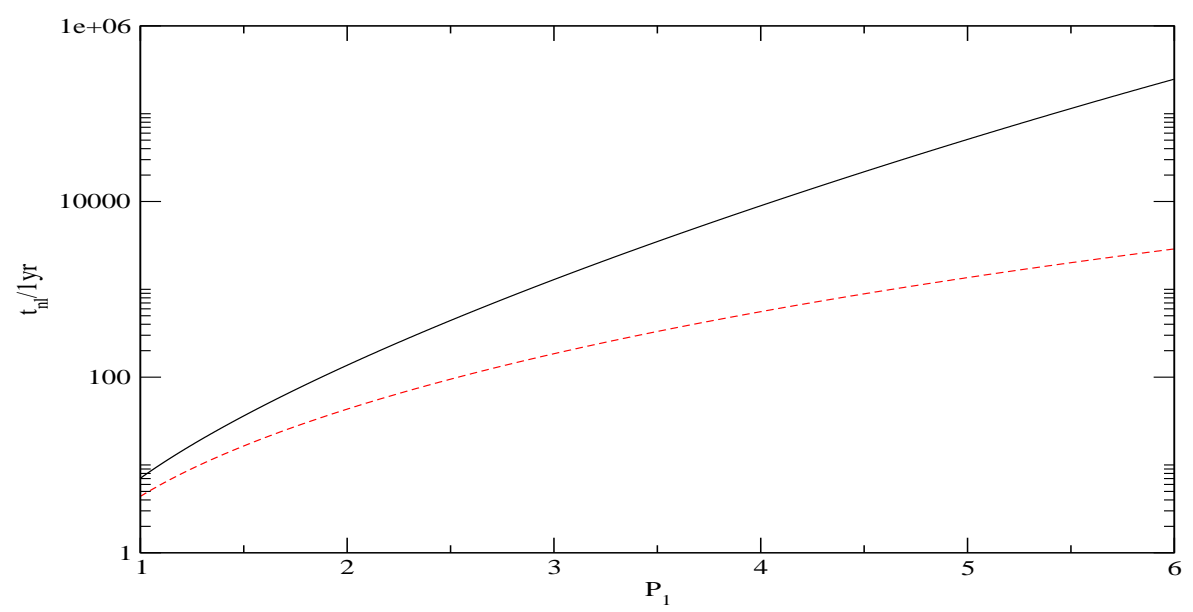

a)

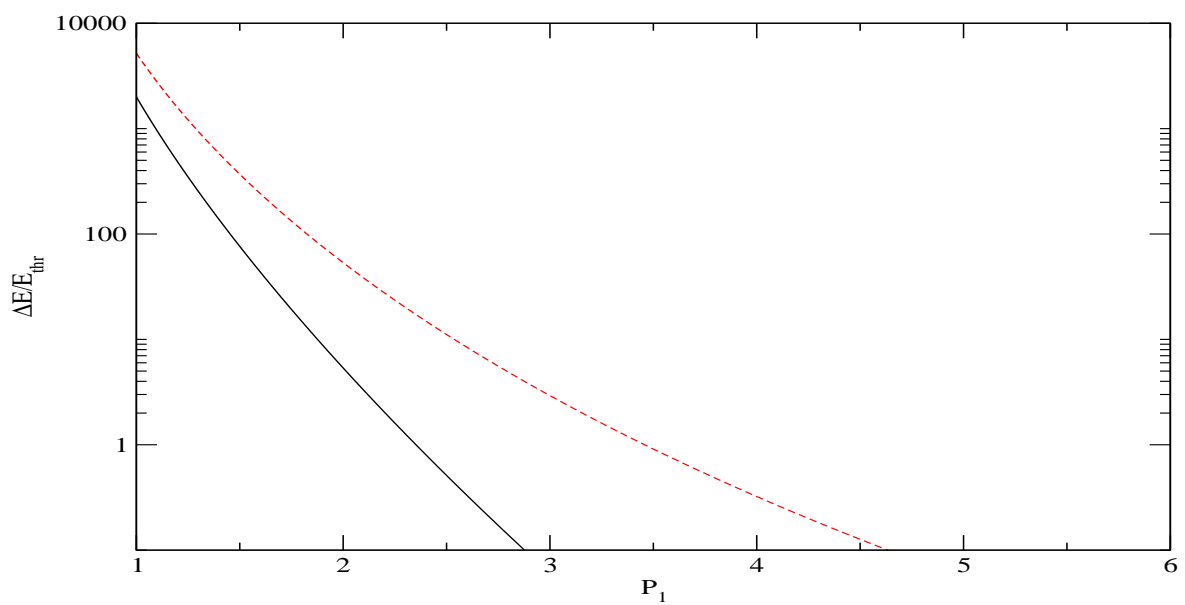

b)

Fig. 7 a) The characteristic time of non-linear dissipation, $t_{n l}$ as a function of the dimensionless orbital period after circularisation, $P_{1}$ for a star with rotational period 1 day. The solid and dashed curves correspond to the prograde and retrograde cases, respectively. b) Ratio of the energy transfer $\Delta E$ to the threshold energy $E_{t h r}$ for the parametric instability to operate. The value of $E_{t h r}$ is taken to be $10^{35} \mathrm{ergs}$, see the text for discussion. 


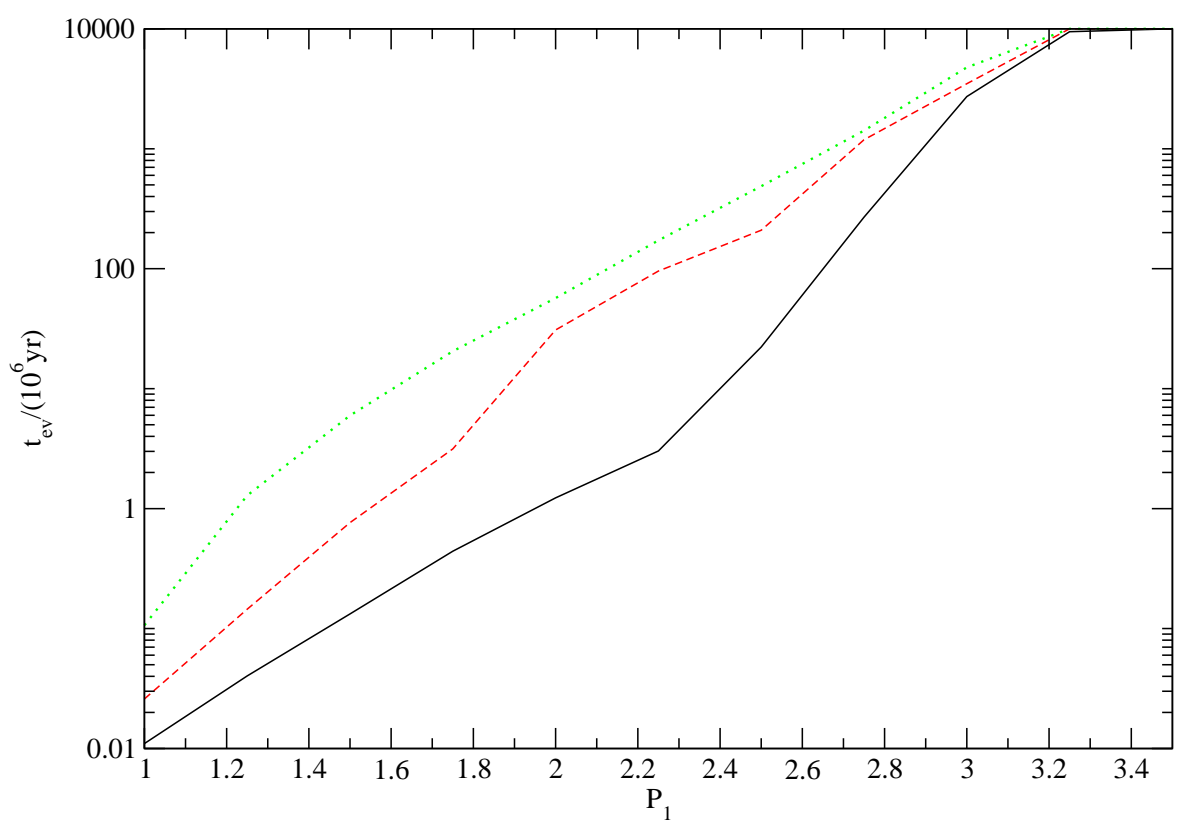

Fig. 8 The evolution time-scale $t_{e v}$ calculated under the assumption that only tides exerted in the star are effective and that the energy transfer is proportional to the attenuation factor given by (59). Note that here we define $t_{e v}$ as a time-scale of evolution of the semi-major axis from its initial value equal taken to be $10 \mathrm{au}$ to a final value taken to be $0.5 \mathrm{au}$. The solid, dashed and dotted curves correspond to retrograde, non-rotating and prograde cases, respectively. The planet's mass $M=1 M_{J}$.

However, in addition to the stochastic instability there is another potentially important process, which may facilitate the orbital evolution at large values of $a$. This is through the so-called non-linear dissipation of the energy $E_{n m}$ stored in the perturbed g-modes of the star (Kumar \& Goodman 1996, hereafter $\mathrm{KG}$ ). This results from the parametric excitation of low frequency high order normal modes through their non-linear interaction with the modes excited by tides. Provided that this energy is larger than a certain threshold value, $E_{t h r}$, the characteristic time scale of dissipation of the energy, $t_{n l}$ is given by the expression $(\mathrm{KG})$

$$
t_{n l} \approx 10 \sqrt{\left(\frac{10^{38} \text { ergs }}{E_{n m}}\right)} y r .
$$


When this time is smaller than than the orbital period $P_{\text {orb }}$ the energy transferred during a single periastron flyby to the normal model can be dissipated in one orbital period, and, accordingly, the expression for $\Delta E$ can be simply added to describe the effect of successive periastron passages. On the other hand, when $f=P_{\text {orb }} / t_{n l} \ll 1$, assuming there is no build up, the energy transferred from the orbit to the star that is actually dissipated should be attenuated by a factor of the order of $f$. A more accurate calculation, which takes into account a possibility of resonances between the orbit and a mode, and therefore a build up transferred energy in the star, gives what we describe as an attenuation factor, $f_{a}$, in the form (see Appendix $\mathrm{C}$ )

$$
f_{a}=(1-\exp (-2 f))\left(1+\exp (-2 f)-2 \exp (-f) \cos \left(2 \pi \omega_{n} / \Omega_{\text {orb }}\right)\right)^{-1},
$$

which is valid for $0<f<\infty$. Here $\omega_{n}$ is a typical value of eigen frequency of a mode excited by tides. For our problem it is of the order of $0.5 \Omega_{*}$. Note that $f_{a} \rightarrow 1$ in the limit $f \rightarrow \infty$ as expected. In order to allow for the weakening of the orbital energy transfer in the limit $f \rightarrow 0$ we adopt $f_{a} \Delta E$ for the energy exchanged per pericentre passage in equation (54) instead of $\Delta E$ itself.

We show the dependence of $t_{n l}$ on $P_{1}$ in Fig. 7(a) for a star with rotational period 1 day and the evolution time scales calculated taking into account the attenuation factor (59) are shown in Fig. 8. As seen from Fig. 7(a) there is a marked difference between the cases of prograde and retrograde motion with the retrograde case corresponding to much smaller values of $t_{n l}$ at given value of $P_{1}$. This can increase the difference between probabilities of tidal capture of planets moving in opposite directions with respect to the stellar rotation. It is also important to note that the threshold value of the energy,$E_{t h r}$, for our case can be estimated to be $E_{t h r} \sim 10^{35} \mathrm{ergs}$. for a star with solar values of mass and radius and rotational period of order of a few day 8 At sufficiently large values of $P_{1}$ (see below) the amount of energy transferred during one periastron flyby of a planet moving on a prograde orbit is significantly smaller than, $E_{t h r}$, making the non-linear dissipation of the mode energy impossible. This can further amplify the difference between the capture probabilities. Interestingly, the values of $t_{n l}$ for retrograde orbits are of the order of the orbital periods of planets with semimajor axes of order $10 \mathrm{au}$ - the scale, where the processes of tidal capture are expected to operate.

In order to calculate $t_{e v}$, as shown in Fig. 8, for simplicity we set $\Delta E_{p l}=0$ in (54). One can see from this figure that when the attenuation effect is taken into account, dynamic tides can explain the initial stages of tidal circularisation up to $P_{1}<3$ in contrast to the simpler case considered in the previous section, where the beginning of effective circularisation is expected up to $P_{1}<4$, see Fig. 5(a). We also checked that typical values of $\Delta E$ used to calculate $t_{e v}$ are approximately equal to $E_{t h r}$ when $P_{1} \sim 3$. Thus, at larger values of $P_{1}$, non-linear dissipation of mode energy is likely to be unimportant.

\footnotetext{
8 In this case KG estimate the value of $E_{t h r}$ to be approximately $10^{48}\left(\omega t_{t h}\right)^{-6 / 5}$ ergs, where $\omega$ is a typical eigen frequency of a mode undergoing parametric instability and $t_{t h}$ is the stellar thermal time. Taking $\omega \approx 0.5 \Omega_{*} \sim 3 \cdot 10^{-4} s^{-1}$ and $t_{t h} \sim 3 \times 10^{7} \mathrm{yr}$ we get $E_{\text {thr }} \sim 10^{35}$ ergs.
} 
The results discussed in this section confirm our previously made assertion that dynamic tides can lead to an efficient decrease of semimajor axis on scales corresponding to tidal capture both as a result of the stochastic instability and the possibility of non-linear dissipation of energy stored in normal modes excited in the star. However, at smaller scales, the evolution of $a$ due to the processes we have discussed clearly stalls, necessitating the consideration of different nonlinear effects, such as wave breaking (e.g. Barker \& Ogilvie (2010), Barker (2011)), operating on normal modes, or other phenomena such as turbulent friction operating on quasi-static tides to be able to describe later stages of orbital evolution.

\section{Discussion}

In this paper we have developed an analytic theory for calculating the tidal response of a slowly rotating star resulting from a fly by of a planetary perturber in a parabolic orbit, with orbital plane at an arbitrary inclination to the stellar equatorial plane. Expressions for the energy transferred to the star were presented in section 3 (see equations (27)-(33)). In order to develop these expressions only the first order corrections to the normal mode eigenfrequencies appropriate to the non rotating star were incorporated. This implicitly assumes that these modes only change slightly and continue to dominate the tidal response in this form. In order to confirm these results numerical solutions of the linear tidal problem were considered in 4 . In these calculations Coriolis forces, but not centrifugal forces, were taken into account. Thus the equilibrium model was spherical. Beyond this no assumption of slow rotation was made. In practice the calculations were done for a polytrope of index $n=3$. Detailed results for prograde encounters, retrograde encounters and encounters with orbital plane perpendicular to the equatorial plane were presented in section 5. These were compared with results obtained from the analytic treatment in section 6. In general the agreement between the two approaches was good. Both agreed that the tidal interaction was significantly more effective for retrograde encounters. The energy exchanged between the orbit and the star was about one order of magnitude larger for retrograde rotation with $\Omega=0.1 \Omega_{*}$ compared to prograde rotation with the same angular velocity when $\eta=4 \sqrt{2}$, with the deviation becoming larger as $\eta$ increased. Nonetheless the numerically obtained results showed an increasing deviation from the analytically determined ones for increasing angular velocity of retrograde rotation. The maximum departure was about a factor of two for $\Omega_{*}=0.25$ and $\eta=4 \sqrt{2}$ increasing to a factor of 2.5 when $\eta$ was increased to 8 . However, due to the very rapid decline in the strength of tidal effects with increasing pericentre distance, we note that such deviations are scarcely significant for practical applications.

The time scale for the initial stage of tidal circularisation inferred from our results for an orbit with initial semi-major axis of $10 \mathrm{au}$ was given in section 6.2 for planets of $1 M_{J}$ and $5 M_{J}$. The rotation of the star, taken to be one solar 
mass, was assumed to follow the Skumanich (1972) law. When tides from both the planet were included, initial circularisation down to circular orbits of periods $<5$ days could be readily accounted for both masses. When the effects of tides in the planet were neglected, our results indicated that tides acting in the star alone could be capable of producing a significant change in the planet's semi-major axis for final periods $<4$ days for both masses. Provided that some additional processes such as quasi-static tides are sufficiently efficient at smaller scales our time scales may give an estimate of a characteristic time of the whole stage of circularisation.

\subsection{Application to the circularisation of Hot Jupiters}

The discovery of Hot Jupiters in orbits highly inclined to the stellar equatorial plane with some orbits even being retrograde has given support to the idea that at least some of these objects were scattered into highly elongated orbits that were the circularised by tidal effects. The process of the excitation of oscillations by tides acting on the planets and the consequent circularisation has been discussed by Ivanov \& Papaloizou (2004, 2007, 2010) and Papaloizou\& Ivanov (2010). Here this work has been extended to consider tides excited in the star. These may be significant because in contrast to tides acting on the planet, they are not equally effective for prograde and retrograde rotation. This feature should be borne in mind when considering the distribution of objects as a function of inclination. Hebrard et al. (2010) point out that of the 37 systems for which the inclination between the orbital and stellar equatorial planes has been measured at the time of writing, about one third are strongly misaligned with the distribution of inclinations being dependent on the planet mass such that retrograde systems only appear for $M<\sim 2.5 M_{J}$. Our results indicate that under the assumptions we made about the stellar angular velocity, circularisation times for prograde and retrograde orbits should differ by a factor of two at least initially, for a 'typical' period after circularisation of the order of four days, and that systems should be seen up to the same limiting period in both types of system. There would thus be some asymmetry produced by the effect of tides in this case though this claim should be confirmed by numerical N-body experiments incorporating planet-planet scattering together with tidal effects that take account of effects resulting from stellar rotation. The asymmetry may be more pronounced for masses $M>\sim 5 M_{J}$. However, at present there are relatively few objects with measured orbital inclinations with respect to the stellar rotation axis measured in this mass range. Future observations may clarify this issue. Similarly results may differ for different histories of the stellar rotation frequency and/or different stellar masses. Winn et all (2010) point out that there is an increase in the fraction of planets on inclined orbits for stars with larger masses. These may have been rotating more rapidly in the past, thus increasing the asymmetry between the prograde and retrograde tidal interactions. 
It is clear that the result for the energy transfer obtained in this paper can also be directly applied to a general situation where two rotating stars of comparable mass with misaligned directions of rotational axes tidally interact with each other. Therefore, our results may be of interest for the problem of tidal capture of stars in young stellar clusters.

Our results can also be generalised in several ways. Firstly, expressions for the transfer of angular momentum can be obtained by a calculation similar to what is made in this paper for the energy transfer. Note that all three components of the angular momentum ( with respect to the coordinate system defined with respect to the stellar rotation axis) are transferred in the case of a general inclined encounter. Secondly, a more realistic stellar model should be used to calculate the eigenfrequencies, overlap integrals and the frequency splitting coefficients $\beta_{r}$. Thirdly, in order to reach better agreement between the theory and the numerical calculations, a better theory of the tidal excitation of high order $g$-modes in a rotating star should, in principle, be developed. In the framework of such a theory one may try to relax the assumption of smallness of the stellar angular velocity in comparison to the mode eigenfrequencies. These issues will be addressed in future investigations.

Acknowledgements We are grateful to M. Nagasawa and A. V. Tutukov for useful remarks. This work was supported by the Science and Technology Facilities Council [grant number ST/G002584/1], by the Dynasty Foundation, by the programme "Research and Research/Teaching staff of Innovative Russia" for 2009 - 2013 years (State Contract No. P 1336 on 2 September 2009) and by RFBR grant 11-02-00244-a.. PBI thanks DAMTP, University of Cambridge for hospitality.

This paper was started when both PBI and JCBP took part in the Isaac Newton Institute programme 'Disks and Planets'.

\section{Appendix A}

\section{Explicit expressions for $d_{m, n}^{(2)}$}

We list below the explicit expressions for the Wigner $d$-functions, which are used in our calculations. We need

$$
d_{2, \pm 2}^{(2)}=\frac{(1 \pm \cos \beta)^{2}}{4}, \quad d_{1, \pm 2}^{(2)}= \pm \frac{\sin \beta(1 \pm \cos \beta)}{2}, \quad d_{0, \pm 2}^{(2)}=d_{ \pm 2,0}^{(2)}=\sqrt{\frac{3}{8}} \sin ^{2} \beta
$$

and the functions obtained from [60] by the rule $d_{-n, \pm 2}^{(2)}=(-1)^{n} d_{n, \mp 2}^{(2)}$ for $n=0,1,2$. Additionally, we use

$$
d_{0,0}^{(2)}=\frac{3 \cos ^{2} \beta-1}{2}, \quad d_{ \pm 1,0}^{(2)}=\mp \sqrt{\frac{3}{2}} \sin \beta \cos \beta .
$$


Appendix B

\section{Transfer of angular momentum during the periastron flyby}

The rate of angular momentum transfer, $\mathbf{j}$, as well as its value of the periastron flyby, $\Delta \mathbf{J}=\int_{-\infty}^{+\infty} d t \dot{\mathbf{J}}$ are determined by the expression

$$
\dot{\mathbf{J}}=\int d^{3} x \rho^{\prime}(\mathbf{r} \times \nabla U),
$$

where $\mathbf{r}$ is the radius vector, and $\rho^{\prime}$ is the density perturbation, which can be found from the continuity equation

$$
\rho^{\prime}=-\nabla \cdot(\rho \xi)=-\sum_{n} \frac{b_{n}(t)}{r}\left(\frac{1}{r} \frac{d}{d r}\left(\rho r^{2} \xi_{r}\right)-6 \rho \xi_{s}\right) Y_{2, n},
$$

where we use equations (15), (16) and the known properties of the spherical functions. Now we substitute equations (3) and (63) in (62), integrate by part the term proportional to $\frac{d}{d r}\left(\rho r^{2} \xi_{r}\right)$ and use (20) to obtain

$$
\dot{\mathbf{J}}=Q \sum_{n, k} A_{n} b_{k} \mathbf{J}_{k, n}, \quad \mathbf{J}_{k, n}=\int d \Omega Y_{2, k}\left(\mathbf{e}_{r} \times r \nabla Y_{2, n}\right),
$$

where integration is performed over the solid angle, and $\mathbf{e}_{r}$ in the unit vector in the radial direction. The quantity $\mathbf{J}_{k, n}$ can be easily evaluated with help of the theory of the vector spherical functions (see e.g. Varshalovich et al 1989) with the result

$$
\mathbf{J}_{k, n}=i(-1)^{k}\left[n \delta_{k,-n} \mathbf{e}_{z}\left(\sqrt{(2-k)(3+k)} \delta_{k,-(n+1)} \mathbf{e}_{-1}-\sqrt{(2+k)(3-k)} \delta_{k, 1-n} \mathbf{e}_{1}\right)\right],
$$

where $\mathbf{e}_{ \pm 1}=\mp \frac{1}{2}\left(\mathbf{e}_{x} \pm i \mathbf{e}_{y}\right)$, and $\mathbf{e}_{\mathbf{x}}, \mathbf{e}_{\mathbf{y}}, \mathbf{e}_{\mathbf{z}}$ are the usual unit vectors associated with the Cartesian coordinates $(x, y, z)$.

In order to calculate $\Delta \mathbf{J}$ we express the quantities $b_{k}$ and $A_{n}$ in (64) through their respective Fourier transforms and integrate the result over time using (7) to get

$$
\Delta \mathbf{J}=Q \sum_{n, k} M_{n, k} \mathbf{J}_{k, n}, \quad M_{n, k}=2 \pi(-1)^{n} \int d \sigma \tilde{b}_{k}(\sigma)\left(\tilde{A}_{-n}(\sigma)\right)^{*} .
$$

Now we substitute (21) in (66) and calculate the integral over $\sigma$ in (66) taking into account only the resonance contribution. The calculation is analogous to the one made for the energy transfer in Section 3 with the result

$$
M_{n, k}=i \frac{\pi^{2}}{\omega} Q\left[(-1)^{n} \tilde{A}_{k}\left(\omega_{k}^{+}\right) \tilde{A}_{-n}\left(\omega_{k}^{+}\right)^{*}-(-1)^{k} \tilde{A}_{n}\left(\omega_{-k}^{+}\right) \tilde{A}_{-k}\left(\omega_{-k}^{+}\right)^{*}\right] .
$$

Now we substitute (65) and (67) in (66) and get the angular momentum transfer in the form

$$
\Delta \mathbf{J}=\Delta J^{z} \mathbf{e}_{z}+\Delta J^{-1} \mathbf{e}_{-1}+\Delta J_{1} \mathbf{e}^{1},
$$

where

$$
\begin{gathered}
\Delta J^{z}=\frac{(\pi Q)^{2}}{\omega} \sum_{k} k\left(\left|\tilde{A}_{k}\left(\omega_{k}^{+}\right)\right|^{2}-\left|\tilde{A}_{-k}\left(\omega_{-k}^{+}\right)\right|^{2}\right) \\
\Delta J^{-1}=\frac{(\pi Q)^{2}}{\omega} \sum_{k} \sqrt{(2-k)(3+k)}\left(\tilde{A}_{k}\left(\omega_{k}^{+}\right) \tilde{A}_{k+1}\left(\omega_{k}^{+}\right)^{*}+\tilde{A}_{-(k+1)}\left(\omega_{-k}^{+}\right) \tilde{A}_{-k}\left(\omega_{-k}^{+}\right)^{*}\right.
\end{gathered}
$$


and $\Delta J^{1}=-\left(\Delta J^{-1}\right)^{*}$. Note that to obtain the latter relation we make a change $k \rightarrow-k$ in the summation series (66) when considering the term proportional to $\mathbf{e}_{1}$. The Cartesian components of the vector of the angular momentum transfer, $\Delta J^{x}$ and $\Delta J^{y}$, can be found from the relations $\Delta J^{x}=\frac{1}{\sqrt{2}}\left(\Delta J^{-1}-\Delta J^{1}\right)$ and $\Delta J^{y}=\frac{i}{\sqrt{2}}\left(\Delta J^{-1}+\Delta J^{1}\right)$. We have

$$
\begin{gathered}
\Delta J^{x}=\frac{(\pi Q)^{2}}{\omega} \sum_{k} \sqrt{(2-k)(3+k)}\left(\tilde{R}_{k}\left(\omega_{k}^{+}\right) \tilde{R}_{k+1}\left(\omega_{k}^{+}\right)+\tilde{I}_{k}\left(\omega_{k}^{+}\right) \tilde{I}_{k+1}\left(\omega_{k}^{+}\right)\right. \\
\left.\quad+\tilde{R}_{-k}\left(\omega_{-k}^{+}\right) \tilde{R}_{-(k+1)}\left(\omega_{-k}^{+}\right)+\tilde{I}_{-k}\left(\omega_{-k}^{+}\right) \tilde{I}_{-(k+1)}\left(\omega_{-k}^{+}\right)\right)
\end{gathered}
$$

and

$$
\begin{aligned}
\Delta J^{y}= & \frac{(\pi Q)^{2}}{\omega} \sum_{k} \sqrt{(2-k)(3+k)}\left(\tilde{I}_{k+1}\left(\omega_{k}^{+}\right) \tilde{R}_{k}\left(\omega_{k}^{+}\right)-\tilde{I}_{k}\left(\omega_{k}^{+}\right) \tilde{R}_{k+1}\left(\omega_{k}^{+}\right)\right. \\
& \left.+\tilde{I}_{-(k+1)}\left(\omega_{-k}^{+}\right) \tilde{R}_{-k}\left(\omega_{-k}^{+}\right)-\tilde{I}_{-k}\left(\omega_{-k}^{+}\right) \tilde{R}_{-(k+1)}\left(\omega_{-k}^{+}\right)\right)
\end{aligned}
$$

where $\tilde{R}_{k}(\sigma)$ and $\tilde{I}_{k}(\sigma)$ are the real and imaginary part of $\tilde{A}_{k}(\sigma)$, respectively, and we remind that $\tilde{A}_{k}(\sigma)$ is defined in equation (14). Equations (69), (71) and (72) allows us to determine all three components of the angular momentum transfer after a tidal encounter.

\section{Appendix C}

\section{Energy transfer from a highly eccentric orbit associated with a nor- mal mode with general damping rate}

Here we consider the energy exchange associated with an orbit of high eccentricity where the mode damping rate may be small. In this case the orbit is assumed to be quasi-periodic and so the system undergoes multiple close approaches in contrast to the single encounter considered in parabolic limit that applies when the mode decay time is shorter than the orbital period. The orbital period is assumed short enough so as to avoid the stochastic regime (see Ivanov \& Papaloizou 2004). We obtain an estimate for the factor by which results for the parabolic case need to be multiplied in order to apply to this case.

We begin by writing down equation (18) governing the response of a single mode amplitude allowing for mode damping (see Ivanov \& Papaloizou 2004) in the form

$$
\ddot{b}_{n}+\omega_{0}^{2} b_{n}+2\left(\gamma_{n}+i \omega_{n}^{1}\right) \dot{b}_{n}+2 i n \gamma_{n} \Omega b_{n}=S, \quad \text { with } \quad S=\int d^{3} x \rho\left(\boldsymbol{\xi}_{n}^{*} \cdot \nabla U\right),
$$

where $\gamma_{n}$ is the mode damping rate. Noting that the problem is very similar to that of a forced, damped harmonic oscillator, we identify the mode energy as

$$
\mathcal{E}_{n}=\frac{1}{2}\left(\left|\dot{b}_{n}\right|^{2}+\omega_{0}^{2}\left|b_{n}\right|^{2}\right)
$$

Its rate of dissipation through mode damping follows from (73) as

$$
\frac{d \mathcal{E}_{n}}{d t}=-2 \gamma_{n}\left|\dot{b}_{n}\right|^{2}-i n \gamma_{n} \Omega\left(b_{n}\left(\dot{b}_{n}\right)^{*}-\left(b_{n}\right)^{*} \dot{b}_{n}\right),
$$

see e.g. Ivanov \& Papaloizou 2004

We consider forcing in a periodic orbit with period $P_{\text {orb }}=2 \pi / \Omega_{\text {orb }}$. The potential $U$ will then have a discrete Fourier series representation in the form

$$
U=\sum_{k=-\infty}^{\infty} \mathcal{U}_{k} \exp \left(-\mathrm{i} k \Omega_{\text {orb }} t\right)
$$


where the Fourier amplitudes are given by

$$
\mathcal{U}_{k}=\frac{\Omega_{\text {orb }}}{2 \pi} \int_{-\pi / \Omega_{\text {orb }}}^{\pi / \Omega_{\text {orb }}} U \exp \left(\mathrm{i} k \Omega_{\text {orb }} t\right) d t .
$$

Note that as the period tends to $\infty$, or $\Omega_{\text {orb }} \rightarrow 0$, with $k \Omega_{\text {orb }}$ remaining finite, $2 \pi \mathcal{U}_{k} / \Omega_{\text {orb }}$ approaches the continuous Fourier transform associated with a parabolic orbit.

Because we consider an essentially linear response, equations (73)-(74) may be applied to each Fourier component separately and then the results combined. Denoting by $S_{k}$, the quantity $S$ evaluated for the corresponding term in the Fourier expansion for $U$, and the associated mode response amplitude by $b_{n k}$, we have

$$
b_{n k}=\frac{S_{k}}{D}, \quad D=\omega_{0}^{2}-k^{2} \Omega_{o r b}^{2}+2 \omega_{n}^{1} k \Omega_{\text {orb }}-2 i \gamma_{n}\left(k \Omega_{\text {orb }}-n \Omega\right) .
$$

As we discuss below only contributions in summation series over $k$, where $\omega_{0} \sim k \Omega_{\text {orb }}$ are important for our purposes. This means that the term proportional to $\gamma_{n} \Omega$ in the expression for $D$ in (78) is much smaller than other terms as long as $\Omega \ll \omega_{0}$. It is neglected below. In the same approximation the term proportional to $\Omega$ in $(75)$ can be neglected as well (in fact these terms could be retained in the analysis but little is gained).

Note that in order to get (78) we assume that the mode damping rate is approximately constant. This is not formally the case when the dissipation is determined by non-linear processes considered in the text and $\gamma_{n}$ is a function of mode amplitude. However, from equation (75) it follows that the characteristic time of mode amplitude decay is long compared with typical periods $1 /\left(k \Omega_{\text {orb }}\right)$ when $\gamma_{n} /\left(k \Omega_{\text {orb }}\right) \ll 1$ which corresponds to the case of interest. Then we can approximately consider $\gamma_{n}$ to be constant and to correspond to the initial rate of of mode decay, $1 / t_{n l}$, when dealing with the Fourier harmonics with high value of $k$ that have frequencies corresponding to those of the excited normal modes.

We now evaluate the rate of energy dissipation by applying equation (74) for each Fourier component and summing over $k$ to give

$$
\frac{d \mathcal{E}_{n}}{d t}=-2 \gamma_{n} \sum_{k=-\infty}^{\infty} \frac{k^{2} \Omega_{o r b}^{2}\left|S_{k}\right|^{2}}{\left[\left[\left(k \Omega_{o r b}-\omega_{n}^{1}-\omega_{0}\right)\left(\omega_{n}^{1}-\omega_{0}-k \Omega_{o r b}\right)+\left(\omega_{n}^{1}\right)^{2}\right]^{2}+4 \gamma_{n}^{2} k^{2} \Omega_{o r b}^{2}\right]}
$$

We may make an estimate for the above sum by noting that for a weakly damped oscillation mode, most of the contribution to the sum is expected to come from values of $k$ corresponding to near resonance between a harmonic of the orbital period and a mode oscillation period. Neglecting the inconsequential quantity $\left(\omega_{n}^{1}\right)^{2}$, which has been assumed to be negligible, resonance occurs when $k$ is such that $k \Omega_{\text {orb }}=\omega_{n}^{1} \pm \omega_{0}$, which in fact corresponds to two modes, one with positive frequency and the other with negative frequency. As the important values of $k$ differ in sign, these can be dealt with separately and the results summed. Without loss of generality, we shall restrict attention to the positive frequency mode for which values of $k$ near $\omega / \Omega_{\text {orb }} \gg 1$ are expected to dominate the sum (we recall that $\omega=\omega_{0}+\omega_{n}^{1}$ ) . Assuming other quantities vary smoothly with $k$, we replace $k$ by $k_{0}$, being the closest integer to $\omega / \Omega_{\text {orb }}$, everywhere except in the first bracket in the denominator of (79) to obtain

$$
\frac{d \mathcal{E}_{n}}{d t}=-\frac{\gamma_{n} \omega^{2}\left|S_{k_{0}}\right|^{2}}{2 \omega_{0}^{2}} \sum_{k=-\infty}^{\infty} \frac{1}{\left[\left(k \Omega_{o r b}-\omega_{n}^{1}-\omega_{0}\right)^{2}+\gamma_{n}^{2}\right]} .
$$

The sum over $k$ is readily performed by standard complex variable techniques to give

$$
\frac{d \mathcal{E}_{n}}{d t}=-\frac{\pi \omega^{2}\left|S_{k_{0}}\right|^{2}}{2 \omega_{0}^{2} \Omega_{\text {orb }}} \mathcal{F}
$$

where

$$
\mathcal{F}=\frac{\left(\exp \left(4 \pi \gamma_{n} / \Omega_{\text {orb }}\right)-1\right)}{\left[\exp \left(4 \pi \gamma_{n} / \Omega_{\text {orb }}\right)+1-2 \exp \left(2 \pi \gamma_{n} / \Omega_{\text {orb }}\right) \cos \left(2 \pi \omega / \Omega_{\text {orb }}\right)\right]} .
$$


To interpret 81 we remark first that when $2 \pi \gamma_{n} / \Omega_{\text {orb }}$ is large corresponding to a damping time shorter than the orbital period, $\mathcal{F}=1$. Then we can integrate 81 over an orbital period to obtain $\Delta\left(\mathcal{E}_{n}\right) \propto\left|S_{k_{0}}\right|^{2} / \Omega_{o r b}^{2}$, which ultimately depends only on the continuous Fourier transform of the perturbing potential. This limit corresponds to a parabolic encounter. More generally $\mathcal{F}$ gives an estimate of the factor by which the energy exchange is modified when the damping time is longer than an orbital period and the motion can be regarded a quasi periodic. When $2 \pi \gamma_{n} / \Omega_{\text {orb }}$ is small, we have

$$
\mathcal{F} \sim \frac{\left(\pi \gamma_{n} / \Omega_{o r b}\right)}{\sin ^{2}\left(\pi \omega / \Omega_{o r b}\right)} .
$$

This factor could be large very close to resonances where $\omega=k \Omega_{\text {orb }}$, for some integer $k$. However, between resonances $\mathcal{F}$ has a minimum value $\gamma_{n} P_{\text {orb }} / 2$, while a reciprocal mean over mode frequencies would suggest a value $\gamma_{n} P_{o r b}$. As orbital evolution very close to resonances is expected to be rapid, the latter values are expected to be more appropriate for estimating evolution times. Recall that for comparison with section 6.2.2 the mode decay rate $\gamma_{n}$ adopted here here should be identified with $1 / t_{n l}$ used there.

\section{References}

1. Allen, A. J., Palmer, P. L., Papaloizou, J. C. B.: A conservative numerical technique for collisionless dynamical systems - Comparison of the radial and circular orbit instabilities. MNRAS, 242, 576 (1990)

2. Barker, A. J., Ogilvie, G. I.: On the tidal evolution of Hot Jupiters on inclined orbits. MNRAS, 395, 2268 (2009)

3. Barker, A. J., Ogilvie, G. I.: On internal wave breaking and tidal dissipation near the centre of a solar-type star. MNRAS, 404, 1849 (2010)

4. Barker, A. J.: Three-dimensional simulations of internal wave breaking and the fate of planets around solar-type stars. MNRAS, to be published, e-print arXiv:1102.0857 (2011)

5. Christensen-Dalsgaard J.:Lecture Notes on Stellar Oscilla- tions, 4th edn. Dept. of Physics and Astronomy, Univ. Aarhus, http://astro.phys.au.dk/223cjcd/oscilnotes/ (1998)

6. Friedman, J. L., Schutz, B. F.: Lagrangian perturbation theory of nonrelativistic fluids. ApJ, 221, 937 (1978)

7. Hebrard, G., Ehrenreich, D., Bouchy, F., Delfosse, X., Moutou, C., Arnold, L., Boisse, I., Bonfils, X., Diaz, R. F., Eggenberger, A., Forveille, T., Lagrange, A-M, Lovis, C., Pepe, F., Perrier, C., Queloz, D., Santerne, A., Santos, N.C., Segransan, D., Udry, S., Vidal-Madjar, A.: The retrograde orbit of the HAT-P-6b exoplanet. A\& A letters, in press

8. Ivanov, P. B., Papaloizou, J. C. B.: On the tidal interaction of massive extra solar planets on highly eccentric orbits. MNRAS, 347, 437 (2004)

9. Ivanov, P. B., Papaloizou, J. C. B.: Dynamic tides in rotating objects: orbital circularisation of extra solar planets for realistic planet models. MNRAS, 376, 682 (2007)

10. Ivanov, P. B., Papaloizou, J. C. B.: Inertial waves in rotating bodies: a WKBJ formalism for inertial waves and a comparison with numerical results. MNRAS, 407, 1631 (2010)

11. Kochanek, C. S.: The dynamical evolution of tidal capture binaries. ApJ, 385, 604 (1992)

12. Kosovichev, A. G.; Novikov, I. D.: Non-linear effects at tidal capture of stars by a massive black hole. I - Incompressible affine model. MNRAS, 258, 715

13. Kumar, P., Goodman, J.: Nonlinear damping of Oscillations in Tidal-Capture Binaries. ApJ, 466, 946 (1996)

14. Lai, D.: Dynamical Tides in Rotating Binary Stars. ApJ, 490, 847 (1997)

15. Lee, H. M., Ostriker, J.P.: Cross sections for tidal capture binary formation and stellar merger. ApJ, 310, 176 (1986)

16. Lin, D. N. C., Papaloizou, J.: On the tidal interaction between protoplanets and the protoplanetary disk. III - Orbital migration of protoplanets. ApJ, 309, 846 (1986)

17. Mardling, R. A.: The Role of Chaos in the Circularization of Tidal Capture Binaries. I. The Chaos Boundary. ApJ, 450, 722 (1995) 
18. Mardling, R. A.: The Role of Chaos in the Circularization of Tidal Capture Binaries. II. Long-Time Evolution. ApJ, 450, 732 (1995)

19. Mardling, R. A., Aarseth, S. J.: Tidal interactions in star cluster simulations. MNRAS, $450,722(2001)$

20. Marchi, S., Ortolani, S., Nagasawa, M., Ida, S.: On the various origins of close-in extrasolar planets. MNRAS, 394, L93 (2009)

21. Nagasawa, M., Ida, S., Bessho, T.: Formation of Hot Planets by a Combination of Planet Scattering, Tidal Circularization, and the Kozai Mechanism. ApJ, 678, 498 (2008)

22. Papaloizou, J. C. B., Ivanov, P. B.: Oscillations of rotating bodies: a self-adjoint formalism applied to dynamic tides and tidal capture. MNRAS, 364L, 66 (2005)

23. Papaloizou, J. C. B., Ivanov, P. B.: Dynamic tides in rotating objects: a numerical investigations of inertial waves in fully convective or baratropic stars and planets. MNRAS, 407, 1631 (2010)

24. Papaloizou, J. C. B., Terquem, C.: Dynamical relaxation and massive extrasolar planets. MNRAS, 325, 221 (2001)

25. Press, W. H., Teukolsky, S. A.: On formation of close binaries by two-body tidal capture. ApJ, 213, 183 (1977)

26. Rasio, F. A., Ford, E. B.: Dynamical instabilities and the formation of extrasolar planetary systems. Science, 274, 954 (1996)

27. Ray, A., Kembhavi, A. K., Antia, H. M.: Evolution of stellar binaries formed by tidal capture. A\&A, 184, 164 (1987)

28. Saio, H.: Rotational and tidal perturbations of nonradial oscillations in a polytropic star. ApJ, 244, 299 (1981)

29. Scholz, A.: Stellar spindown: From the ONC to the Sun. COOL STARS, STELLAR SYSTEMS AND THE SUN: Proc. of the 15th Cambridge Workshop on Cool Stars, Stellar Systems and the Sun. AIP Conference Proc., 1094, 61 (2009)

30. Skumanich, A.: Time Scales for CA II Emission Decay, Rotational Braking, and Lithium Depletion. ApJ, 171, 565 (1972)

31. Varshalovich, D. A., Moskalev, A. N., Khersonskii, V. K.:Quantum Theory of Angular Momentum. World Scientific Publishing Co Pte Ltd, Singapore (1988)

32. Weidenschilling, S. J., Marzari, F.: Gravitational scattering as a possible origin for giant planets at small stellar distances. Nature, 384, 619 (1996)

33. Winn, J. N., Fabrycky, D., Albrecht, S., Johnson, J. A.: Hot Stars with Hot Jupiters Have High Obliquities. ApJ, 718, L145 (2010) 